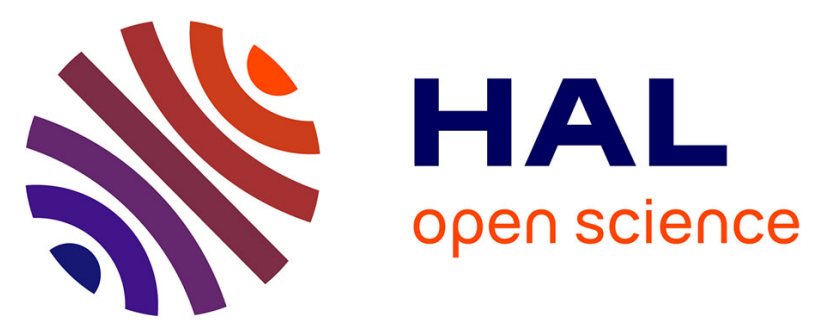

\title{
Quasi-static Free-Boundary Equilibrium of Toroidal Plasma with CEDRES++: Computational Methods and Applications
}

Holger Heumann, Jacques Blum, Cedric Boulbe, Blaise Faugeras, Gael Selig, P. Hertout, Eric Nardon, Jean-Marc Ané, Sylvain Brémond, Virginie

Grandgirard

\section{To cite this version:}

Holger Heumann, Jacques Blum, Cedric Boulbe, Blaise Faugeras, Gael Selig, et al.. Quasi-static Free-Boundary Equilibrium of Toroidal Plasma with CEDRES++: Computational Methods and Applications. Journal of Plasma Physics, 2015, pp.35. 10.1017/S0022377814001251 . hal-01088772

\section{HAL Id: hal-01088772 \\ https://inria.hal.science/hal-01088772}

Submitted on 1 Dec 2014

HAL is a multi-disciplinary open access archive for the deposit and dissemination of scientific research documents, whether they are published or not. The documents may come from teaching and research institutions in France or abroad, or from public or private research centers.
L'archive ouverte pluridisciplinaire HAL, est destinée au dépôt et à la diffusion de documents scientifiques de niveau recherche, publiés ou non, émanant des établissements d'enseignement et de recherche français ou étrangers, des laboratoires publics ou privés. 


\title{
Quasi-static Free-Boundary Equilibrium of Toroidal Plasma with CEDRES++: Computational Methods and Applications
}

\author{
H. HEUMA N N $\mathbf{N}^{1} \dagger$, J. B L U M $\mathbf{M}^{1}$, C. B O U L B E $\mathbf{E}^{1}$, \\ B. F A U G E R A $S^{1}$, G. SEL I G ${ }^{1}$, \\ J. - M. A N É ${ }^{2}$, S. BRÉ M O N D ${ }^{2}$, V. GRA N D G I R A R D ${ }^{2}$, \\ P. HERTOU T ${ }^{2}$, E. NARD O N ${ }^{2}$ \\ ${ }^{1}$ TEAM CASTOR, INRIA and Université de Nice Sophia Antipolis, Parc Valrose, 06108 Nice \\ Cedex 02, France \\ ${ }^{2}$ CEA, IRFM, F-13108 Saint-Paul-lez-Durance, France
}

(Received 25 November 2014)

\begin{abstract}
We present a comprehensive survey of the various computational methods in CEDRES++ for finding equilibria of toroidal plasma. Our focus is on free-boundary plasma equilibria, where either poloidal field coil currents or the temporal evolution of voltages in poloidal field circuit systems are given data. Centered around a piecewise linear finite element representation of the poloidal flux map, our approach allows in large parts the use of established numerical schemes. The coupling of a finite element method and a boundary element method gives consistent numerical solutions for equilibrium problems in unbounded domains. We formulate a new Newton method for the discretized nonlinear problem to tackle the various non-linearities, including the free plasma boundary. The Newton method guarantees fast convergence and is the main building block for the inverse equilibrium problems that we can handle in CEDRES++ as well. The inverse problems aim at finding either poloidal field coil currents that ensure a desired shape and position of the plasma or at finding the evolution of the voltages in the poloidal field circuit systems that ensure a prescribed evolution of the plasma shape and position. We provide equilibrium simulations for the tokamaks ITER and WEST to illustrate the performance of CEDRES++ and its application areas.
\end{abstract}

\section{Introduction}

Computer codes that address the equilibrium of toroidal plasmas are central tools in tokamak fusion science. They are essential, both for detailed simulations with sophisticated magnetohydrodynamic (MHD) models as well as for experimenters that need to control real tokamak reactors. Detailed MHD simulations, which model the plasma on very short timescales, are used to study the various effects of turbulence and instability. They rely on a given plasma equilibrium as initial condition. Experimenters use equilibrium codes to set up discharge scenarios, to study breakdowns and disruptions, or to design the layout of new machines. They also use such codes, in connection with transport codes (Hinton \& Hazeltine 1976, Hirshman \& Jardin 1979, Artaud et al. 2010, Coster et al. 2010: Parail et al. 2013), to design and validate plasma feedback controller for real tokamak machines and to verify the feasibility of scenarios in terms of operational limits (e.g. coil currents or forces).

$\dagger$ Email address for correspondence: holger.heumann@inria.fr 
Hence, equilibrium codes are essential tools for tokamak scientists, and applicants expect a certain degree of maturity and robustness. In the design of discharge scenarios or in the validation of feedback controller, for example, a robust, fast and automated computation of equilibria allows to shift the focus of research towards the difficulties of coupling with complex physics or improved control algorithms. CEDRES ++ deals with equilibrium problems that are related to a quasi-static description of plasma evolution, which asserts balance of forces at each instant of time. A code that treats such quasi-static free-boundary equilibrium problems needs to solve non-linear elliptic or parabolic problems with non-linear source terms representing the current density profile, that vanishes outside the unknown free boundary of the plasma. The computational challenges in the design of free-boundary equilibrium codes are a problem setting in an unbounded domain with a non-linearity due the current density profile in the unknown plasma domain and the non-linear magnetic permeability if the reactor has ferromagnetic structures.

The simulation on the unbounded domain can be reduced to computations on a finite domain thanks to analytical Green's functions (Lackner 1976). The numerical solution on the finite interior domain is coupled through boundary conditions to the Green's function representation of the solution in the exterior domain. This approach is today fairly standard in many other application areas such as electromagnetics (Hiptmair 2003, Zhao et al. 2006) or elasticity (Costabel \& Stephan 1990; Bielak \& MacCamy 1991; Stephan 1992). The boundary element method (Chen \& Zhou |1992 |Nédélec |2001) is the name of this general framework. The boundary element method reduces problems on unbounded domains to problems on boundaries, that can then be coupled to any numerical method for the interior of a bounded domain.

The non-linearity due to the current profile in the unknown plasma domain poses the major difficulties according to our experience. It is a peculiarity of plasma equilibrium problems, that the domain of the plasma is an unknown. Speaking differently, the boundary of the plasma is a free boundary, defined either by a contact with a limiter which prevents the plasma from touching the vacuum vessel, or defined as being a separatrix in the case of a poloidal divertor configuration. On top of this fairly unusual kind of nonlinearity, also the current profile in the plasma itself is a non-linear function. Moreover, in the so-called iron transformer tokamaks, a third type of non-linearity appears due to the non-linear magnetic permeability. All these non-linearities will require some iterations towards the numerical solution. Simple fixed-point iterations usually suffer from very slow convergence or even fail to converge, which made researchers move towards Newton-type methods. The latter use the information of gradients, sometimes also referred to as sensitivities, to speed up the convergence, and they can converge in cases where fixed-point iterations don't converge - a very important example is vertically unstable plasmas.

There are basically two different families of solution methods for axisymmetric plasma equilibrium problems. The first family are the so-called flux or Lagrangian coordinate methods, determining the localization of level lines that have equidistant flux-values (Lao et al. 1985, 1981; Ling \& Jardin 1985: Turkington et al. 1993: Gruber et al. 1987; Degtyarev \& Drozdov 1991; DeLucia et al. | 1980 Jardin et al. 1986; DeLucia et al. 1980; Degtyarev \& Drozdov 1985) (see also (Jardin 2010, section 5.5)). A second family of methods uses standard finite difference methods on rectangular grids (Feneberg \& Lackner 1973, Helton \& Wang 1978; Johnson et al. 1979; Lackner 1976) or finite element methods on triangular grids (Blum et al. 1981). The main difference between most methods of both of these families is the treatment of the so-called fixed boundary equilibrium problem, i.e. a problem where the plasma domain is known. The computational issues related to the unknown boundary have received less attention.

The CEDRES ++ code uses a finite element formulation for the axisymmetric free- 
boundary equilibrium problem in the interior domain. This allows first, for standard, well established coupling methods to the boundary element formulation on the exterior domain (Albanese et al. 1986). Second, we can derive a perfect Newton method, that uses the information about all non-linearities, e.g. also those related to the free-boundary setting. We consider this to be the most distinctive feature of CEDRES++ among many other equilibrium codes. Up to our knowledge there is no other equilibrium solver that uses this information to speed up the convergence. Furthermore, accurate derivatives are vital for inverse free-boundary equilibrium problems, which aim at finding the values of control parameters that ensure that the plasma attains a certain desired state, i.e. shape or position. Inverse free-boundary equilibrium problems are formulated as constrained optimization problems and only accurately computed derivatives can guarantee that the optimization algorithms find indeed the optimum. For the moment, CEDRES ++ uses linear Lagrangian elements, which due to the low regularity of the solution, seem to be the obvious choice. We would like to refer to Section 5 for a general discussion on this topic.

CEDRES ++ inherits the basic ideas of the free-boundary equilibrium codes SCED (Blum et al. 1981) and Proteus (Albanese et al. 1987) but relies on object oriented and modular programming principles. CEDRES ++ uses well established and tested external modules for e.g. mesh generation (Shewchuk 1996), linear algebra (Renard \& Pommier 2014) and algebraic solver (Davis 2011). The very first conception of CEDRES++, that used the same methods as SCED and Proteus, was developed in (Grandgirard 1999). Various simulations with this old version of CEDRES++ are reported in (Grandgirard 1999) and (Hertout et al. 2011).

The current version of CEDRES++ contains a new module that, when coupled to a transport code, simulates a quasi-statically evolving equilibrium: the classical GradShafranov equation, a non-linear elliptic partial differential equation, is satisfied at each instant of time. This mode assumes that the evolution of voltages in poloidal field circuits and the non-linearities in plasma current profile are known. The new mode is referred to as the evolution mode as opposed to the static mode that takes poloidal field coil currents and the current density as input. Within the new evolution mode, we solve the full parabolic partial differential equation system. We do not have to estimate the nonlinear mutual inductance of the plasma with the electromagnetic reactor components as the approach in (Albanese \& Villone 1998) and (Ariola \& Pironti 2008, Chapter 2) would require. All the dynamics of the plasma core related to resistive diffusion of magnetic flux and transport of particle density and temperatures, are supposed to be treated by external tools and are not subject of this report. We refer to (Falchetto et al. 2014) for the coupling of CEDRES++ (Couplage Equilibre Diffusion Résistive pour l'Etude des Scénarios i.e. Coupling of Equilibrium and Resistive Diffusion for the Evaluation of Scenarios) with the transport code ETS (Coster et al. 2010). CEDRES++ is also coupled to the transport code CRONOS (Artaud et al.|2010). The evolution mode used with prescribed evolution of the current profile is also a good practical approach for vertical stability studies, where the timescale of interest is much shorter than the current diffusion timescale of the plasma.

Further, CEDRES ++ can solve inverse free-boundary equilibrium problems. The inverse problem in the static mode aims at finding poloidal field coil currents that ensure a desired shape and position of the plasma. The inverse problem in the evolution mode aims at finding the evolution of the voltages in the poloidal field circuits that ensure a prescribed evolution of the plasma shape and position. We use standard algorithms for constrained optimization to solve the inverse problems. Therefore it will be straightforward to add in the near future further constraints, such as constraints on the flux 
consumption or the currents in the coils. In table1 we summarize the basic CEDRES++ modes and their areas of application.

Previous implementations of the Newton method in SCED (Blum et al. 1981) and Proteus (Albanese et al. 1987) relied on the discretization of a Newton method formulated on a continuous level. It is not clear, whether this formulation remains valid for equilibria with plasma boundaries in the case of a poloidal divertor configuration. The distinctive new feature of CEDRES++ is a Newton method, that solves the discretized non-linear equations. Our new approach has more rigorous mathematical foundations and is supposed to have slightly faster convergence. Moreover, it is only this new approach, which guarantees that the optimization algorithms for solving the inverse problems converge to the correct solution. Section 3 gives more explanations on that.

The users of CEDRES++ do not need to know about details of the algorithms and the parameters. CEDRES++ is a robust, fast and accurate and an easily usable tool. CEDRES ++ focuses for the moment on the solution of the so called axisymmetric freeboundary plasma equilibrium with isotropic pressure and without flow. The assumption of perfect axial symmetry is a common model reduction in many equilibrium applications and the treatment of $3 D$ plasma equilibria (Park et al. 1999 , Hirshman \& Betancourt 1991) requires still a lot of computational power. We are planning to include in the near future numerical methods for plasma equilibria with flow and plasma equilibria with anisotropic pressure (Grad 1967, Maschke \& Perrin 1984; Goedbloed \& Lifschitz 1997; Zwingmann et al. 2001; Guazzotto et al. |2004; Cooper et al. 2009; Pustovitov 2010; Fitzgerald et al. 2013). Toroidal equilibria with anisotropic pressure and flow are an active area of research that will benefit from our contribution to the computation of free-boundary equilibria. CEDRES++ is not considered to be used as a so-called equilibrium reconstruction code (Hofmann \& Tonetti 1988; Lao et al. 1990; 1 Mc Carthy et al. 1999 , Blum et al. 2012), which relies on measurements during the discharge to compute the magnetic fields and estimates of current profiles and other characteristics of plasma equilibria.

The outline of the article is the following: In the first section we recall briefly the basic equations that describe the free-boundary plasma equilibrium in a tokamak and state the four main problems that can be solved with CEDRES++. The subsequent section contains detailed descriptions of the various numerical methods that are implemented in CEDRES++. This is followed by a short section containing tests for the numerical validation and various application examples.

\section{Quasi-Static Free-Boundary Equilibrium of Toroidal Plasma}

The essential equations for describing plasma equilibrium in a tokamak are force balance, the solenoidal condition and Ampère's law

$$
\operatorname{grad} p=\mathbf{J} \times \mathbf{B}, \quad \operatorname{div} \mathbf{B}=0, \quad \operatorname{curl} \frac{1}{\mu} \mathbf{B}=\mathbf{J},
$$

where $p$ is the plasma kinetic pressure, $\mathbf{B}$ is the magnetic field, $\mathbf{J}$ is the current density and $\mu$ the magnetic permeability. In the quasi-static approximation these static equations are augmented by Faraday's law

$$
-\partial_{t} \mathbf{B}=\operatorname{curl} \mathbf{E},
$$

with $\mathbf{E}$ the electric field, and by Ohm's laws in plasma, coils and passive structures.

For the calculations in CEDRES++ we will differentiate between static problems and evolution problems, where the keyword static indicates that the equations do not give 


\begin{tabular}{|l|l|l|}
\hline | CEDRES + mode & functionality & application areas \\
\hline \hline static, direct & $\begin{array}{l}\text { simulates plasma equilibria for } \\
\text { given poloidal field coil currents }\end{array}$ & $\begin{array}{l}\text { reference equilibria, initial condi- } \\
\text { tions for short timescale plasma } \\
\text { models }\end{array}$ \\
\hline static, inverse & $\begin{array}{l}\text { finds the poloidal field coil cur- } \\
\text { rents which allow the best match } \\
\text { with a given plasma shape at a } \\
\text { fixed time }\end{array}$ & preparation of scenarios \\
\hline evolution, direct & $\begin{array}{l}\text { simulates the quasi-static evolu- } \\
\text { tion of plasma equilibrium for } \\
\text { given poloidal field circuit volt- } \\
\text { ages }\end{array}$ & $\begin{array}{l}\text { coupling to transport codes, de- } \\
\text { sign and test environment for } \\
\text { feedback controller, study of } \\
\text { breakdowns and disruptions. }\end{array}$ \\
\hline evolution, inverse & $\begin{array}{l}\text { finds optimal poloidal field circuit } \\
\text { voltages and desired evolution of } \\
\text { the plasma }\end{array}$ & $\begin{array}{l}\text { feedforward control for discharge } \\
\text { scenario optimization. }\end{array}$ \\
\hline
\end{tabular}

\section{TABLE 1.}

a time-varying solution. The static problems and the evolution problems are treated by CEDRES++ static mode and evolution mode, respectively. Force balance, solenoidal condition and Ampère's law in 2.1) yield the static problem we will introduce in detail in Section 2.1. while the evolution problems introduced afterwards in Section 2.3, take also into account the Faraday's and Ohm's law in the poloidal field coils and in the passive structures. All the dynamics due to Faraday's and Ohm's laws in the plasma, as well as the dynamics related to transport of heat and particles are supposed to be treated by external tools.

Under the common assumption of perfect axial symmetry, it is convenient to put (2.1) and the quasi-static approximation of Maxwell's equations in a cylindrical coordinate system $(r, \varphi, z)$ and to consider only a meridian section of the tokamak. Then, Faraday's and Ampère's laws decouple into corresponding laws in the toroidal direction and the poloidal plane and give rise to the toroidal and poloidal Poynting theorems:

$$
\operatorname{div} \frac{\mathbf{B}_{t o r} \times \mathbf{E}_{p o l}}{\mu}=\mathbf{J}_{p o l} \cdot \mathbf{E}_{p o l}+\frac{1}{2 \mu} \partial_{t} \mathbf{B}_{t o r}^{2}
$$

and

$$
\operatorname{div} \frac{\mathbf{B}_{p o l} \times \mathbf{E}_{t o r}}{\mu}=\mathbf{J}_{t o r} \cdot \mathbf{E}_{t o r}+\frac{1}{2 \mu} \partial_{t} \mathbf{B}_{p o l}^{2},
$$

where the subscripts tor and pol indicate the toroidal and poloidal components of the fields. Poynting theorems can be used to check the conservation of energy in the simulation of quasi-static plasma equilibria, thus providing a global control of the accuracy.

We introduce $\Omega_{\infty}=[0, \infty] \times[-\infty, \infty]$, the positive half plane, to denote the meridian plane that contains the tokamak centered at the origin. The geometry of the tokamak determines the various subdomains (see Figure1): 


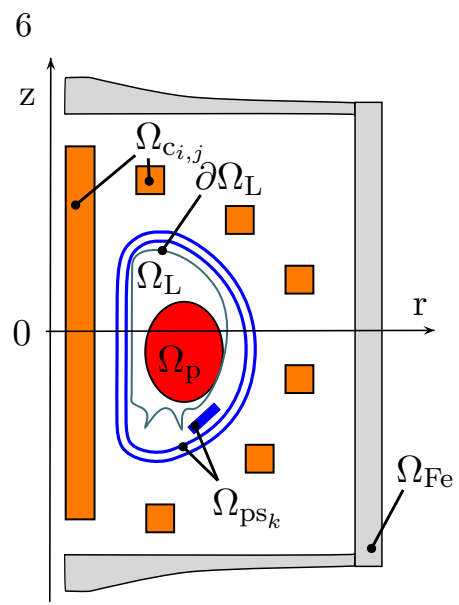

FiguRE 1. Left: Geometric description of the tokamak in the poloidal plane. Middle and right: Sketch for characteristic plasma shapes. The plasma boundary touches the limiter (middle) or the plasma is enclosed by a flux line that goes through an X-point (right).

- the domain $\Omega_{\mathrm{Fe}} \subset \Omega_{\infty}$ corresponds to those parts that are made of iron; for an air-transformer tokamak $\Omega_{\mathrm{Fe}}=\emptyset$;

- the domains $\Omega_{\mathrm{c}_{i, j}} \subset \Omega_{\infty}, 1 \leqslant i \leqslant L, 1 \leqslant j \leqslant N_{i}$, correspond to the $\sum_{i=1}^{L} N_{i}=N$ poloidal field coils. The coils are grouped into $L$ poloidal field circuits and the $i$ th circuit contains $N_{i}$ coils. The intersection of the $j$ th coil in the $i$ th circuit with the poloidal plane is $\Omega_{c_{i, j}}$, and it has $n_{i, j}$ wire turns, total resistance $R_{i, j}$ and cross section area $S_{i, j}$;

- the domains $\Omega_{\mathrm{ps}_{k}} \subset \Omega_{\infty}, k=1, \ldots, N_{\mathrm{ps}}$ corresponding to $N_{\mathrm{ps}}$ passive structures with conductivity $\sigma_{k}$;

- the domain $\Omega_{\mathrm{L}} \subset \Omega_{\infty}$, bounded by the limiter, corresponds to the domain which is accessible by the plasma;

- the domain $\Omega_{\mathrm{p}} \subset \Omega_{\mathrm{L}}$, is the domain covered by the plasma.

The classical primal unknowns for toroidal plasma equilibria described by (2.1) are the poloidal magnetic flux $\psi=\psi(r, z)$, the pressure $p$ and the diamagnetic function $f$. The poloidal magnetic flux $\psi:=r \mathbf{A} \cdot \mathbf{e}_{\varphi}$ is the scaled toroidal component of the vector potential $\mathbf{A}$, i.e. $\mathbf{B}=\operatorname{curl} \mathbf{A}$ and $\mathbf{e}_{\varphi}$ the unit vector for $\varphi$. The diamagnetic function $f=r \mathbf{B} \cdot \mathbf{e}_{\varphi}$ is the scaled toroidal component of the magnetic field. It can be shown that both the pressure $p$ and the diamagnetic function $f$ are constant on $\psi$-isolines, i.e. $p=p(\psi)$ and $f=f(\psi)$. We refer to standard text books, e.g. (Freidberg 1987), (Blum 1987), (Wesson 2004), (Goedbloed \& Poedts 2004), (Goedbloed et al.|2010) and (Jardin 2010) for the details and state in the following paragraphs only the final equations describing the static and evolution problems solved in CEDRES++.

\subsection{Direct Static Problem}

Force balance, solenoidal condition and Ampère's law in 2.1 yield in axisymmetric configuration the following set of equations:

$$
\begin{aligned}
-\nabla \cdot\left(\frac{1}{\mu r} \nabla \psi\right) & = \begin{cases}r p^{\prime}(\psi)+\frac{1}{\mu_{0} r} f f^{\prime}(\psi) & \text { in } \Omega_{\mathrm{p}}(\psi) ; \\
\frac{I_{i, j}}{S_{i, j}} & \text { in } \Omega_{c_{i, j}} ; \\
0 & \text { elsewhere },\end{cases} \\
\psi(0, z) & =0 ; \quad \lim _{\|(r, z)\| \rightarrow+\infty} \psi(r, z)=0 ;
\end{aligned}
$$


where $\nabla$ is the gradient in the two dimensions $(r, z), I_{i, j}$ is the total current (in Ampère turns) in the $j$-th coil of the $i$ th circuit and $\mu$ is a functional of $\psi$

$$
\mu= \begin{cases}\mu_{\mathrm{Fe}}\left(|\nabla \psi|^{2} r^{-2}\right) & \text { in } \Omega_{\mathrm{Fe}} \\ \mu_{0} & \text { elsewhere } .\end{cases}
$$

with $\mu_{0}$ the constant magnetic permeability of vacuum and $\mu_{\mathrm{Fe}}$ the non-linear magnetic permeability of iron. Here again, we would like to stress that the plasma domain $\Omega_{\mathrm{p}}(\psi)$ is an unknown, which depends non-linearly on the magnetic flux $\psi$ : the plasma domain $\Omega_{\mathrm{p}}(\psi)$ is a functional of the poloidal flux $\psi$. The different characteristic shapes of $\Omega_{\mathrm{p}}(\psi)$ are illustrated in Figure 1 . the boundary of $\Omega_{\mathrm{p}}(\psi)$ either touches the boundary of $\Omega_{\mathrm{L}}$ (limiter case) or the boundary contains one or more saddle points of $\psi$ (divertor configuration). The saddle points of $\psi$, denoted by $\left(r_{\mathrm{X}}, z_{\mathrm{X}}\right)=\left(r_{\mathrm{X}}(\psi), z_{\mathrm{X}}(\psi)\right)$, are called X-points of $\psi$. The plasma domain $\Omega_{\mathrm{p}}(\psi)$ is the largest subdomain of $\Omega_{\mathrm{L}}$ bounded by a closed $\psi$ isoline in $\Omega_{\mathrm{L}}$ and containing the magnetic axis $\left(r_{\mathrm{ax}}, z_{\mathrm{ax}}\right)$. The magnetic axis is the point $\left(r_{\mathrm{ax}}, z_{\mathrm{ax}}\right)=\left(r_{\mathrm{ax}}(\psi), z_{\mathrm{ax}}(\psi)\right)$, where $\psi$ has its global maximum in $\Omega_{\mathrm{L}}$. For convenience, we introduce also the coordinates $\left(r_{\mathrm{bnd}}, z_{\mathrm{bnd}}\right)=\left(r_{\mathrm{bnd}}(\psi), z_{\mathrm{bnd}}(\psi)\right)$ of the point that determines the plasma boundary. $\left(r_{\mathrm{bnd}}, z_{\mathrm{bnd}}\right)$ is either an X-point of $\psi$ or the contact point with the limiter $\partial \Omega_{\mathrm{L}}$.

The equation 2.5 in the plasma domain, i.e.

$$
-\frac{\partial}{\partial r}\left(\frac{1}{\mu_{0} r} \frac{\partial \psi}{\partial r}\right)-\frac{\partial}{\partial z}\left(\frac{1}{\mu_{0} r} \frac{\partial \psi}{\partial z}\right)=r p^{\prime}(\psi)+\frac{1}{\mu_{0} r} f f^{\prime}(\psi),
$$

is the celebrated Grad-Shafranov-Schlüter equation (Grad \& Rubin|1958: Shafranov 1958, Lüst \& Schlüter 1957). The domain of $p^{\prime}$ and $f f^{\prime}$ is the interval $\left[\psi_{\text {bnd }}, \psi_{\text {ax }}\right]$ with the scalar values $\psi_{\mathrm{ax}}$ and $\psi_{\mathrm{bnd}}$ being the flux values at the magnetic axis and at the boundary of the plasma:

$$
\begin{aligned}
\psi_{\text {ax }}(\psi) & :=\psi\left(r_{\text {ax }}(\psi), z_{\text {ax }}(\psi)\right), \\
\psi_{\text {bnd }}(\psi) & :=\psi\left(r_{\text {bnd }}(\psi), z_{\text {bnd }}(\psi)\right) .
\end{aligned}
$$

The two functions $p^{\prime}$ and $f f^{\prime}$ and the currents $I_{i, j}$ in the coils are not determined by the model (2.5) and have to be supplied as data. Since the domain of $p^{\prime}$ and $f f^{\prime}$ depends on the poloidal flux itself, it is more practical to supply those profiles as functions of the normalized poloidal flux $\psi_{\mathrm{N}}(r, z)$ :

$$
\psi_{\mathrm{N}}(r, z)=\frac{\psi(r, z)-\psi_{\mathrm{ax}}(\psi)}{\psi_{\mathrm{bnd}}(\psi)-\psi_{\mathrm{ax}}(\psi)} .
$$

These two functions, subsequently termed $S_{p^{\prime}}$ and $S_{f f^{\prime}}$, have, independently of $\psi$, a fixed domain $[0,1]$.

Further, in many applications, one assumes that the current profile, i.e. the function $r S_{p^{\prime}}+\frac{1}{\mu_{0} r} S_{f f^{\prime}}$, is only known up to some scaling constant $\lambda$. In those cases the set of equations in 2.5 has to be augmented by an additional equation that matches the scaling with the given total plasma current $I_{\mathrm{P}}$.

Let us state the two problems that we will consider in the following.

Problem 1 (Direct Static). Let $S_{p^{\prime}}:[0,1] \rightarrow \mathbb{R}$ and $S_{f f^{\prime}}:[0,1] \rightarrow \mathbb{R}$ be two known functions and let the currents $I_{i, j}$ in the coils be given. We want to find the $\psi$ such that 2.5. holds with $p^{\prime}(\psi)=S_{p^{\prime}}\left(\psi_{\mathrm{N}}\right)$ and $f f^{\prime}(\psi)=S_{f f^{\prime}}\left(\psi_{\mathrm{N}}\right)$.

Problem 2 (Direct static, with given plasma current $I_{\mathrm{P}}$ ). Let $S_{p^{\prime}}:[0,1] \rightarrow \mathbb{R}$ and $S_{f f^{\prime}}:[0,1] \rightarrow \mathbb{R}$ be two known functions and let the currents $I_{i, j}$ in the coils be given. 
Additionally we assume that the total plasma current $I_{\mathrm{P}}$ is given. We want to find $\psi$ and $\lambda$ such that 2.5. holds with $p^{\prime}(\psi)=\lambda S_{p^{\prime}}\left(\psi_{\mathrm{N}}\right)$ and $f f^{\prime}(\psi)=\lambda S_{f f^{\prime}}\left(\psi_{\mathrm{N}}\right)$ together with

$$
I_{\mathrm{P}}=\lambda \int_{\Omega_{\mathrm{p}}(\psi)}\left(r S_{p^{\prime}}\left(\psi_{\mathrm{N}}(r, z)\right)+\frac{1}{\mu_{0} r} S_{f f^{\prime}}\left(\psi_{\mathrm{N}}(r, z)\right)\right) d r d z .
$$

The functions $S_{p^{\prime}}$ and $S_{f f^{\prime}}$ are usually given as piecewise polynomial functions. Another frequent a priori model is

$$
S_{p^{\prime}}\left(\psi_{\mathrm{N}}\right)=\frac{\beta}{r_{0}}\left(1-\psi_{\mathrm{N}}^{\alpha}\right)^{\gamma}, \quad S_{f f^{\prime}}\left(\psi_{\mathrm{N}}\right)=(1-\beta) \mu_{0} r_{0}\left(1-\psi_{\mathrm{N}}^{\alpha}\right)^{\gamma},
$$

with $r_{0}$ the major radius of the vacuum chamber and $\alpha, \beta, \gamma \in \mathbb{R}$ given parameters. We refer to (Luxon \& Brown 1982) for a physical interpretation of these parameters. The parameter $\beta$ is related to the poloidal beta, whereas $\alpha$ and $\gamma$ describe the peakage of the current profile.

\subsection{Inverse Static Problem}

The direct problem in the previous section computes a free-boundary equilibrium for given coil currents. In many applications, in particular in the area of tokamak operation, the inverse problem is equally relevant: What are the currents that give a certain desired shape to the plasma? A popular approach to answer such a question is its formulation as an optimal control problem. The currents $I_{i}$ in the poloidal field coils are the control variables and the magnetic flux map $\psi$ describing the equilibrium is the controlled variable. Then we introduce a cost function for the magnetic flux $\psi$ and the coil currents $I_{i}$ penalizing the deviation from a desired plasma shape and position, and we minimize this cost function under the constraint that the magnetic flux $\psi$ and the currents in the coils solve the equilibrium problem 2.5. A regularization term ensures well-posedness of the inverse problem. Here again, the current profile in the plasma is supposed to be known data.

In CEDRES++ we prescribe a plasma state by a desired plasma boundary $\Gamma_{\text {desi }}$. Let $\Gamma_{\text {desi }} \subset \Omega_{\mathrm{L}}$ denote a closed line, contained in the domain $\Omega_{\mathrm{L}}$ that is either smooth and touches the limiter at one point or has at least one corner. The former case prescribes a desired plasma boundary that touches the limiter. The latter case aims at a plasma with X-point lying entirely in the interior of $\Omega_{\mathrm{L}}$. Further let $\left(r_{\text {desi }}, z_{\text {desi }}\right) \in \Gamma_{\text {desi }}$ and $\left(r_{1}, z_{1}\right), \ldots\left(r_{N_{\text {desi }}}, z_{N_{\text {desi }}}\right) \in \Gamma_{\text {desi }}$ be $N_{\text {desi }}+1$ points on that line. We define a quadratic cost functional $K(\psi)$ that evaluates to zero if $\Gamma_{\text {desi }}$ is a $\psi$-isoline, i.e. if $\psi$ is constant on $\Gamma_{\text {desi }}$ :

$$
K(\psi):=\frac{1}{2} \sum_{i=1}^{N_{\text {desi }}}\left(\psi\left(r_{i}, z_{i}\right)-\psi\left(r_{\text {desi }}, z_{\text {desi }}\right)\right)^{2} .
$$

Another quadratic cost functional, that will serve as regularization, is

$$
R\left(I_{1,1}, \ldots, I_{L, N_{L}}\right):=\sum_{i=1}^{L} \sum_{j=1}^{N_{i}} \frac{w_{i, j}}{2} I_{i, j}^{2} .
$$

The coefficients $w_{i, j} \geqslant 0$ are called regularization weights.

Let us state the two inverse problems that we will consider in the following.

Problem 3 (Inverse static). Let $S_{p^{\prime}}:[0,1] \rightarrow \mathbb{R}$ and $S_{f f^{\prime}}:[0,1] \rightarrow \mathbb{R}$ be two 
known functions. We solve the following minimization problem:

$$
\min _{\psi, I_{1,1}, \ldots I_{L, N_{L}}} K(\psi)+R\left(I_{1,1}, \ldots I_{L, N_{L}}\right) \quad \text { subject to } 2.5
$$

with $p^{\prime}(\psi)=S_{p^{\prime}}\left(\psi_{\mathrm{N}}\right)$ and $f f^{\prime}(\psi)=S_{f f^{\prime}}\left(\psi_{\mathrm{N}}\right)$.

Problem 4 (Inverse static, with given Plasma Current $I_{\mathrm{P}}$ ). Let $S_{p^{\prime}}:[0,1] \rightarrow$ $\mathbb{R}$ and $S_{f f^{\prime}}:[0,1] \rightarrow \mathbb{R}$ be two known functions and assume additionally that the total plasma current $I_{\mathrm{P}}$ is given. We solve the following minimization problem:

$$
\min _{\lambda, \psi, I_{1,1}, \ldots I_{L, N_{L}}} K(\psi)+R\left(I_{1,1}, \ldots I_{L, N_{L}}\right) \quad \text { subject to 2.5 and 2.10 }
$$

with $p^{\prime}(\psi)=\lambda S_{p^{\prime}}\left(\psi_{\mathrm{N}}\right)$ and $f f^{\prime}(\psi)=\lambda S_{f f^{\prime}}\left(\psi_{\mathrm{N}}\right)$.

Clearly, it is also possible to define other cost functions forcing the plasma to have other characteristics. CEDRES ++ can be easily extended in this direction. Furthermore it is possible to add both equality and inequality constraints. We are planning to include for example upper and lower bounds on the currents and the forces in the coils.

Another class of inverse problems related to static equilibrium, appears in real time tokamak control. There, it is important to reconstruct both the plasma boundary as well as the current profile functions $p^{\prime}$ and $f f^{\prime}$ in the plasma from external measurements. Frequent and fast prediction of the current state of the plasma in the tokamak machine are essential information for feedback control system. Hence, the computational challenges in solving these inverse problems are much different, and lead to the development of a separate class of equilibrium codes (Hofmann \& Tonetti 1988, Lao et al. 1990; Mc Carthy et al. 1999, Blum et al. 2012).

\subsection{Direct Evolution Problem}

In contrast to the static problems, the evolution problems in CEDRES ++ take also into account the Faraday's and Ohm's laws in the poloidal field coils and in the passive structures. The dynamics due to Faraday's and Ohm's law in the plasma, as well as the dynamics related to transport of heat and particles are supposed to be treated by external tools. Alternatively, one can prescribe the profiles $S_{p^{\prime}}$ and $S_{f f^{\prime}}$ as functions of time. The Poynting theorems 2.4 and 2.3) could provide a global mean to check whether the coupling between CEDRES ++ and such external tools is accurate. However, due to discretization, one needs to resort to integrated versions of the Poynting theorems for the accuracy check. Later, in section 3.6, we will present detailed formulas of such integrated Poynting theorems.

The $N$ poloidal field coils are gathered into $L$ poloidal field circuits which contain in total $M$ supplies. Each of the $L$ poloidal field circuits contains a subset of the $N$ coils and a subset of the $M$ supplies. We denote by $\vec{I}_{i}$ the vector of size $M_{i}+N_{i}$ which contains the currents at the $M_{i}$ supplies and in the $N_{i}$ coils of the circuit with index $i, 1 \leqslant i \leqslant L$. The circuit equations in the $i$ th circuit can be written in the form:

$$
\vec{I}_{i}=\boldsymbol{S}_{i} \vec{V}_{i}+\boldsymbol{R}_{i} \vec{\Psi}_{i}\left(\partial_{t} \psi\right),
$$

where the matrices $\boldsymbol{S}_{i} \in \mathbb{R}^{\left(M_{i}+N_{i}\right) \times M_{i}}$ and $\boldsymbol{R}_{i} \in \mathbb{R}^{\left(M_{i}+N_{i}\right) \times N_{i}}$ depend on the wire turns

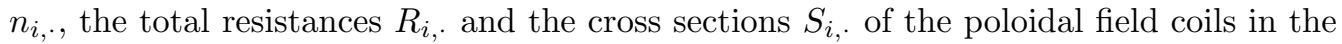
circuit $i$ and on the topology of the circuit. Details on the computation of matrices $\boldsymbol{S}_{i}$ and $\boldsymbol{R}_{i}$ are given in Appendix (A). The vectors $\vec{V}_{i} \in \mathbb{R}^{M_{i}}$ contain the voltages applied to the supplies, and the vectors $\vec{\Psi}_{i}(\psi) \in \mathbb{R}^{N_{i}}$ are $\vec{\Psi}_{i}(\psi)=\left(\int_{\Omega_{c_{i, 1}}} \psi d r d z, \ldots, \int_{\Omega_{c_{i, N_{i}}}} \psi d r d z\right)^{T}$. 
In the case of a simple circuit composed of one supply connected to the coil $\Omega_{c_{i, 1}}$ the equation (2.16) writes

$$
I_{i, 1}=\frac{n_{i, 1} V_{i, 1}(t)}{R_{i, 1}}-2 \pi \frac{n_{i, 1}^{2}}{R_{i, 1} S_{i, 1}} \int_{\Omega_{c_{i, 1}}} \frac{\partial \psi}{\partial t} d r d z .
$$

The free-boundary equilibrium problem on the time interval $[0, T]$ for the time dependent poloidal flux $\psi=\psi(t)=\psi(r, z, t)$ is:

$$
\begin{aligned}
-\nabla \cdot\left(\frac{1}{\mu r} \nabla \psi\right) & = \begin{cases}r p^{\prime}(\psi, t)+\frac{1}{\mu_{0} r} f f^{\prime}(\psi, t) & \text { in } \Omega_{\mathrm{p}}(\psi) ; \\
S_{i, j}^{-1}\left(\boldsymbol{S}_{i} \vec{V}_{i}+\boldsymbol{R}_{i} \vec{\Psi}_{i}\left(\partial_{t} \psi\right)\right)_{j} & \text { in } \Omega_{\mathrm{c}_{i, j}}, 1 \leqslant i \leqslant L, 1 \leqslant j \leqslant N_{i} \\
-\frac{\sigma_{k}}{r} \frac{\partial \psi}{\partial t} & \text { in } \Omega_{\mathrm{ps}_{k}} ; \\
0 & \text { elsewhere }\end{cases} \\
\psi(0, z, t) & =0 ; \quad \lim _{\|(r, z)\| \rightarrow+\infty} \psi(r, z, t)=0 ; \\
\psi(r, z, 0) & =\psi_{0}(r, z),
\end{aligned}
$$

The equation in the passive structures $\Omega_{\mathrm{ps}_{k}}$ is deduced from Ohm's law and Faraday's law, $\sigma_{k}$ being the equivalent axi-symmetric conductivity.

Problem 5 (Evolution, Direct). Let $S_{p^{\prime}}:[0,1] \times[0, T] \rightarrow \mathbb{R}$ and $S_{f f^{\prime}}:[0,1] \times$ $[0, T] \rightarrow \mathbb{R}$ be two known functions. Let the evolution of the voltages $\vec{V}_{1}(t), \ldots, \vec{V}_{L}(t)$ in the poloidal field circuits and the initial data $\psi_{0}$ be given. We want to find the evolution of $\psi(t)$ such that 2.17) holds with $p^{\prime}(\psi(t), t)=\lambda S_{p^{\prime}}\left(\psi_{\mathrm{N}}(t), t\right)$ and $f f^{\prime}(\psi(t), t)=\lambda S_{f f^{\prime}}\left(\psi_{\mathrm{N}}(t), t\right)$.

Problem 6 (evolution, with given plasma current $I_{\mathrm{P}}(t)$, Direct). Let $S_{p^{\prime}}$ : $[0,1] \times[0, T] \rightarrow \mathbb{R}$ and $S_{f f^{\prime}}:[0,1] \times[0, T] \rightarrow \mathbb{R}$ be two known functions. Let the evolution of the voltages $\vec{V}_{1}(t), \ldots, \vec{V}_{L}(t)$ in the poloidal field circuits and the initial data $\psi_{0}$ be given. Additionally we assume that the evolution of the total plasma current $I_{\mathrm{P}}(t)$ is given. We want to find the evolution of $\psi(t)$ and $\lambda(t)$ such that $(2.17)$ holds with $p^{\prime}(\psi(t), t)=$ $\lambda S_{p^{\prime}}\left(\psi_{\mathrm{N}}(t), t\right)$ and $f f^{\prime}(\psi(t), t)=\lambda S_{f f^{\prime}}\left(\psi_{\mathrm{N}}(t), t\right)$ together with

$$
I_{\mathrm{P}}(t)=\lambda(t) \int_{\Omega_{\mathrm{p}}(\psi(t))}\left(r S_{p^{\prime}}\left(\psi_{\mathrm{N}}(r, z, t), t\right)+\frac{1}{\mu_{0} r} S_{f f^{\prime}}\left(\psi_{\mathrm{N}}(r, z, t), t\right)\right) d r d z
$$

To model a consistent quasi-static evolution of plasma equilibrium the equations in 2.17) have to be augmented by the diffusion of density, temperature and magnetic flux. In that case, both functions $S_{p^{\prime}}$ and $S_{f f^{\prime}}$ in the Problems 5 and 6 appear as unknowns of the full system of equations.

\subsection{Inverse Evolution Problem}

The inverse evolution problem is the problem of determining external voltages such that the evolution of the plasma has certain prescribed properties. We will state this problem again as an optimal control problem.

Let $\Gamma_{\text {desi }}(t) \subset \Omega_{\mathrm{L}}$ denote the evolution of a closed line, contained in the domain $\Omega_{\mathrm{L}}$ that is either smooth and touches the limiter at one point or has at least one corner. The former case prescribes a desired plasma boundary that touches the limiter. The latter case aims at a plasma with X-point that is entirely in the interior of $\Omega_{\mathrm{L}}$. Further let $\left(r_{\text {desi }}(t), z_{\text {desi }}(t)\right) \in \Gamma_{\text {desi }}(t)$ and $\left(r_{1}(t), z_{1}(t)\right), \ldots,\left(r_{N_{\text {desi }}}(t), z_{N_{\text {desi }}}(t)\right) \in \Gamma_{\text {desi }}(t)$ be 
$N_{\text {desi }}+1$ points on that line. We define a quadratic functional $K(\psi)$ that evaluates to zero if $\Gamma_{\text {desi }}(t)$ is an $\psi(t)$-isoline, i.e. if $\psi(t)$ is constant on $\Gamma_{\text {desi }}(t)$ :

$$
K(\psi(t)):=\frac{1}{2} \int_{0}^{T}\left(\sum_{i=1}^{N_{\mathrm{desi}}}\left(\psi\left(r_{i}(t), z_{i}(t), t\right)-\psi\left(r_{\mathrm{desi}}(t), z_{\mathrm{desi}}(t), t\right)\right)^{2}\right) d t
$$

Another functional, that will serve as regularization, is

$$
R\left(\vec{V}_{1}(t), \ldots, \vec{V}_{L}(t)\right):=\sum_{i=1}^{L} \frac{w_{i}}{2} \int_{0}^{T} \vec{V}_{i}(t) \cdot \vec{V}_{i}(t) d t .
$$

It penalizes the strength of the voltages $\vec{V}_{i}$ and represents the energetic cost in the coil system. The coefficients $w_{i} \geqslant 0$ are called regularization weights.

Problem 7 (Evolution, inverse). Let $S_{p^{\prime}}:[0,1] \times[0, T] \rightarrow \mathbb{R}$ and $S_{f f^{\prime}}:[0,1] \times \mathbb{R} \rightarrow$ $\mathbb{R}$ be two known functions. We solve the following minimization problem:

$$
\min _{\psi(t), \vec{V}_{1}(t), \ldots \vec{V}_{L}(t)} K(\psi(t))+R\left(\vec{V}_{1}(t), \ldots \vec{V}_{L}(t)\right) \quad \text { subject to 2.17 }
$$

with $p^{\prime}(\psi(t), t)=S_{p^{\prime}}\left(\psi_{\mathrm{N}}(t), t\right)$ and $f f^{\prime}(\psi(t))=S_{f f^{\prime}}\left(\psi_{\mathrm{N}}(t), t\right)$.

Problem 8 (Evolution, with given plasma current $I_{\mathrm{P}}(t)$, inverse). Let $S_{p^{\prime}}$ : $[0,1] \times[0, T] \rightarrow \mathbb{R}$ and $S_{f f^{\prime}}:[0,1] \times \mathbb{R} \rightarrow \mathbb{R}$ be two known functions. Additionally we assume that the evolution of the total plasma current $I_{\mathrm{P}}(t)$ is given. We solve the following minimization problem:

$$
\begin{aligned}
& \min _{\lambda(t), \psi(t), \vec{V}_{1}(t), \ldots \vec{V}_{L}(t)} K(\psi(t))+R\left(\vec{V}_{1}(t), \ldots \vec{V}_{L}(t)\right) \quad \text { subject to 2.17) and 2.18) } \\
& \text { with } p^{\prime}(\psi(t), t)=\lambda(t) S_{p^{\prime}}\left(\psi_{\mathrm{N}}(t), t\right) \text { and } f f^{\prime}(\psi(t))=\lambda(t) S_{f f^{\prime}}\left(\psi_{\mathrm{N}}(t), t\right) .
\end{aligned}
$$

\section{Computational Methods and Applications}

The main challenges for solving the Problems 1 8 numerically are their formulation on an infinite domain, the non-linear right-hand side, the non-linear permeability in iron and the non-linearity due to the free plasma boundary. In the following we will use finite element methods (Ciarlet 1978) to discretize the problems 1 1 , and we will see that this approach is flexible enough to tackle all those challenges at once. First, finite element methods are favored approximation methods due to their flexibility on domains with complex geometry. Second, they allow for a straight forward implementation of Newton methods to handle the strong non-linearities related to the free boundary setting. The convergence speed of such Newton methods is superior to the convergence speed of fixed-point approaches that are otherwise applied for such kind of problems. As a variational formulation is the starting point for any finite element method the section starts with the variational formulations of Problems 1, 2, 5 and 6. The subsequent paragraph on the spatial discretization, a standard finite element method with linear Lagrangian basis functions on triangles, focuses mainly on the special treatment of the free plasma boundary. It gives the important formulas, required to derive the new Newton method afterwards. Having these Newton methods at hand, it is straightforward to tackle the inverse problems. The overview on the computational methods finishes with two paragraphs describing the interfaces of CEDRES ++ for the coupling with transport codes and presenting volume integrated Poynting theorems. 


\subsection{Variational Formulation on the Truncated Domain}

We chose a semi-circle $\Gamma$ of radius $\rho_{\Gamma}$ surrounding the iron domain $\Omega_{\mathrm{Fe}}$, the coil domains $\Omega_{c_{i, j}}$ and the passive structures domain $\Omega_{\mathrm{ps}_{k}}$. The truncated domain, we use for our computations, is the domain $\Omega \subset \Omega_{\infty}$ having the boundary $\partial \Omega=\Gamma \cup \Gamma_{r=0}$, where $\Gamma_{r=0}:=\{(r, z), r=0\}$. The variational formulations of Problems 1, 2, 5 and 6 use the following Sobolev space:

$$
V:=\left\{\psi: \Omega \rightarrow \mathbb{R}, \int_{\Omega} \psi^{2} r d r d z<\infty, \int_{\Omega}(\nabla \psi)^{2} r^{-1} d r d z<\infty, \psi_{\mid \Gamma_{r=0}}=0\right\} \cap C^{0}(\bar{\Omega})
$$

and are obtained by multiplying equations in 1, 2, 5 and 6 by testfunctions $\xi \in V$ and integrating by parts over $\Omega$. They are called the variational formulations since they are the Euler equations of the mininization of the energy. Then we define

- two mappings A : $V \times V \rightarrow \mathbb{R}$ and $\mathrm{J}_{\mathrm{p}}: V \times V \rightarrow \mathbb{R}$ that are linear in the last argument:

$$
\begin{aligned}
\mathrm{A}(\psi, \xi) & :=\int_{\Omega} \frac{1}{\mu(\psi) r} \nabla \psi \cdot \nabla \xi d r d z \\
\mathrm{~J}_{\mathrm{p}}(\psi, \xi) & :=\int_{\Omega_{\mathrm{p}}(\psi)}\left(r p^{\prime}(\psi)+\frac{1}{\mu_{0} r} f f^{\prime}(\psi)\right) \xi d r d z
\end{aligned}
$$

- two bilinear forms $\mathrm{j}^{\mathrm{ps}}, \mathrm{j}^{\mathrm{c}}: V \times V \rightarrow \mathbb{R}$

$$
\begin{aligned}
\mathrm{j}^{\mathrm{ps}}(\psi, \xi) & :=-\sum_{i=1}^{N_{\mathrm{ps}}} \int_{\Omega_{\mathrm{ps}_{k}}} \frac{\sigma_{k}}{r} \psi \xi d r d z \\
\mathrm{j}^{\mathrm{c}}(\psi, \xi) & :=\sum_{i=1}^{L} \sum_{j=1}^{N_{i}} S_{i, j}^{-1}\left(\boldsymbol{R}_{i} \vec{\Psi}_{i}(\psi)\right)_{j} \int_{\Omega_{\mathrm{c}_{i, j}}} \xi d r d z
\end{aligned}
$$

- $N$ bilinear mappings $\ell_{i, j}: \mathbb{R} \times V \rightarrow \mathbb{R}$ :

$$
\ell_{i, j}(I, \xi):=S_{i, j}^{-1} I \int_{\Omega_{c_{i, j}}} \xi d r d z
$$

- a bilinear form c : $V \times V \rightarrow \mathbb{R}$, accounting for the boundary conditions at infinity (Albanese et al. 1986):

$$
\begin{aligned}
\mathrm{c}(\psi, \xi):= & \frac{1}{\mu_{0}} \int_{\Gamma} \psi\left(\mathbf{P}_{1}\right) N\left(\mathbf{P}_{1}\right) \xi\left(\mathbf{P}_{1}\right) d S_{1} \\
& +\frac{1}{2 \mu_{0}} \int_{\Gamma} \int_{\Gamma}\left(\psi\left(\mathbf{P}_{1}\right)-\psi\left(\mathbf{P}_{2}\right)\right) M\left(\mathbf{P}_{1}, \mathbf{P}_{2}\right)\left(\xi\left(\mathbf{P}_{1}\right)-\xi\left(\mathbf{P}_{2}\right)\right) d S_{1} d S_{2} .
\end{aligned}
$$

with

$$
\begin{aligned}
M\left(\mathbf{P}_{1}, \mathbf{P}_{2}\right) & =\frac{k_{\mathbf{P}_{1}, \mathbf{P}_{2}}}{2 \pi\left(r_{1} r_{2}\right)^{\frac{3}{2}}}\left(\frac{2-k_{\mathbf{P}_{1}, \mathbf{P}_{2}}^{2}}{2-2 k_{\mathbf{P}_{1}, \mathbf{P}_{2}}^{2}} E\left(k_{\mathbf{P}_{1}, \mathbf{P}_{2}}\right)-K\left(k_{\mathbf{P}_{1}, \mathbf{P}_{2}}\right)\right) \\
N\left(\mathbf{P}_{1}\right) & =\frac{1}{r_{1}}\left(\frac{1}{\delta_{+}}+\frac{1}{\delta_{-}}-\frac{1}{\rho_{\Gamma}}\right) \text { and } \delta_{ \pm}=\sqrt{r_{1}^{2}+\left(\rho_{\Gamma} \pm z_{1}\right)^{2}},
\end{aligned}
$$

where $\mathbf{P}_{i}=\left(r_{i}, z_{i}\right)$ and $K$ and $E$ the complete elliptic integrals of first and second kind, respectively and

$$
k_{\mathbf{P}_{j}, \mathbf{P}_{k}}=\sqrt{\frac{4 r_{j} r_{k}}{\left(r_{j}+r_{k}\right)^{2}+\left(z_{j}-z_{k}\right)^{2}}} .
$$


We refer to (Grandgirard 1999, Chapter 2.4) for the details of the derivation. The bilinear form $\mathrm{c}(\cdot, \cdot)$ follows basically from the so called uncoupling procedure in (Gatica \& Hsiao 1995) for the usual coupling of boundary integral and finite element methods. In our case, it can be shown that for all $\mathbf{P}_{1}, \mathbf{P}_{2}$ the integral term $\left(\psi\left(\mathbf{P}_{1}\right)-\psi\left(\mathbf{P}_{2}\right)\right) M\left(\mathbf{P}_{1}, \mathbf{P}_{2}\right)\left(\xi\left(\mathbf{P}_{1}\right)-\right.$ $\left.\xi\left(\mathbf{P}_{2}\right)\right)$ remains bounded. The Green's function that is used in the derivation of the boundary integral method for our problem was used earlier in finite difference methods for the Grad-Shafranov-Schlüter equations (Lackner 1976).

We derive the following variational formulations of the direct Problems 1 and 2

Variational Formulation 9 (Static). Let $S_{p^{\prime}}:[0,1] \rightarrow \mathbb{R}$ and $S_{f_{f^{\prime}}}:[0,1] \rightarrow \mathbb{R}$ be two known functions and let the currents $I_{i, j}$ in the coils be given. We set $p^{\prime}(\psi)=S_{p^{\prime}}\left(\psi_{\mathrm{N}}\right)$ and $f f^{\prime}(\psi)=S_{f f^{\prime}}\left(\psi_{\mathrm{N}}\right)$ in (3.2). We want to find $\psi \in V$ such that

$$
\mathrm{A}(\psi, \xi)-\mathrm{J}_{\mathrm{p}}(\psi, \xi)+\mathrm{c}(\psi, \xi)=\sum_{i=1}^{L} \sum_{j=1}^{N_{i}} \ell_{i, j}\left(I_{i, j}, \xi\right)
$$

holds for all $\xi \in V$.

Variational Formulation 10 (Static, with given plasma Current $I_{\mathrm{P}}$ ). Let $S_{p^{\prime}}:[0,1] \rightarrow \mathbb{R}$ and $S_{f f^{\prime}}:[0,1] \rightarrow \mathbb{R}$ be two known functions and let the currents $I_{i, j}$ in the coils be given. We set $p^{\prime}(\psi)=S_{p^{\prime}}\left(\psi_{\mathrm{N}}\right)$ and $f f^{\prime}(\psi)=S_{f f^{\prime}}\left(\psi_{\mathrm{N}}\right)$ in (3.2). Additionally we assume that the total plasma current $I_{\mathrm{P}}$ is given. We want to find $\psi \in V$ and $\lambda \in \mathbb{R}$ such that

$$
\begin{aligned}
\mathrm{A}(\psi, \xi)-\lambda \mathrm{J}_{\mathrm{p}}(\psi, \xi)+\mathrm{c}(\psi, \xi) & =\sum_{i=1}^{L} \sum_{j=1}^{N_{i}} \ell_{i, j}\left(I_{i, j}, \xi\right), \\
I_{\mathrm{p}}-\lambda \mathrm{J}_{\mathrm{p}}(\psi, 1) & =0
\end{aligned}
$$

holds for all $\xi \in V$.

The variational formulation of the evolution problems 5 and 6 is based on an implicit Euler timestepping scheme $0:=t_{0}<t_{0}+\Delta t_{1}=t_{1}<\ldots t_{n-1}+\Delta t_{n}=t_{n}=T$. Other choices are possible. Since we will anyway employ only low order spatial discretization, the implicit Euler is the obvious choice.

Variational Formulation 11 (Evolution). Let $S_{p^{\prime}}:[0,1] \times[0, T] \rightarrow \mathbb{R}$ and $S_{f f^{\prime}}:[0,1] \times[0, T] \rightarrow \mathbb{R}$ be two known functions. Let the evolution of the voltages $\vec{V}_{1}(t), \ldots, \vec{V}_{L}(t)$ in the poloidal field circuits and the initial data $\psi_{0}$ be given. We set $p^{\prime}(\psi)=S_{p^{\prime}}\left(\psi_{\mathrm{N}}(t), t\right)$ and $f f^{\prime}(\psi)=S_{f f^{\prime}}\left(\psi_{\mathrm{N}}(t), t\right)$ in $(3.2)$. We want to find $\psi^{k} \in V$ approximating $\psi\left(t_{k}\right)$ such that

$$
\begin{gathered}
\Delta t_{k} \mathrm{~A}\left(\psi^{k}, \xi\right)-\Delta t_{k} \mathrm{~J}_{\mathrm{p}}^{k}\left(\psi^{k}, \xi\right)-\mathrm{j}^{\mathrm{ps}}\left(\psi^{k}, \xi\right)-\mathrm{j}^{\mathrm{c}}\left(\psi^{k}, \xi\right)+\Delta t_{k} \mathrm{c}\left(\psi^{k}, \xi\right) \\
=\Delta t_{k} \sum_{i=1}^{L} \sum_{j=1}^{N_{i}} \ell_{i, j}\left(\left(\boldsymbol{S}_{i} \vec{V}_{i}\left(t_{k}\right)\right)_{j}, \xi\right)-\mathrm{j}^{\mathrm{ps}}\left(\psi^{k-1}, \xi\right)-\mathrm{j}^{\mathrm{c}}\left(\psi^{k-1}, \xi\right), \\
\psi^{0}=\psi_{0}
\end{gathered}
$$

holds for all $\xi \in V$ with $\mathrm{J}_{\mathrm{p}}^{k}(\cdot, \cdot)=\mathrm{J}_{\mathrm{p}}(\cdot, \cdot)_{\mid t=t_{k}}$.

Variational Formulation 12 (Evolution, with Given Plasma CURREnt $I_{\mathrm{P}}(t)$ ). Let $S_{p^{\prime}}:[0,1] \times[0, T] \rightarrow \mathbb{R}$ and $S_{f f^{\prime}}:[0,1] \times[0, T] \rightarrow \mathbb{R}$ be two known functions. Let the evolution of the voltages $\vec{V}_{1}(t), \ldots, \vec{V}_{L}(t)$ in the poloidal field circuits and the initial 
data $\psi_{0}$ be given. We set $p^{\prime}(\psi)=S_{p^{\prime}}\left(\psi_{\mathrm{N}}(t), t\right)$ and $f f^{\prime}(\psi)=S_{f f^{\prime}}\left(\psi_{\mathrm{N}}(t), t\right)$ in (3.2). Additionally we assume that the evolution of the total plasma current $I_{\mathrm{P}}(t)$ is given. We want to find $\psi^{k} \in V$ and $\lambda^{k} \in \mathbb{R}$ approximating $\psi\left(t_{k}\right)$ and $\lambda\left(t_{k}\right)$ such that

$$
\begin{gathered}
\Delta t_{k} \mathrm{~A}\left(\psi^{k}, \xi\right)-\Delta t_{k} \lambda^{k} \mathrm{~J}_{\mathrm{p}}^{k}\left(\psi^{k}, \xi\right)-\mathrm{j}^{\mathrm{ps}}\left(\psi^{k}, \xi\right)-\mathrm{j}^{\mathrm{c}}\left(\psi^{k}, \xi\right)+\Delta t_{k} \mathrm{c}\left(\psi^{k}, \xi\right) \\
=\Delta t_{k} \sum_{i=1}^{L} \sum_{j=1}^{N_{i}} \ell_{i, j}\left(\left(\boldsymbol{S}_{i} \vec{V}_{i}\left(t_{k}\right)\right)_{j}, \xi\right)-\mathrm{j}^{\mathrm{ps}}\left(\psi^{k-1}, \xi\right)-\mathrm{j}^{\mathrm{c}}\left(\psi^{k-1}, \xi\right), \\
I_{\mathrm{p}}\left(t_{k}\right)-\lambda^{k} \mathrm{~J}_{\mathrm{p}}^{k}\left(\psi^{k}, 1\right)=0, \quad \psi^{0}=\psi_{0} .
\end{gathered}
$$

holds for all $\xi \in V$ with $\mathrm{J}_{\mathrm{p}}^{k}(\cdot, \cdot)=\mathrm{J}_{\mathrm{p}}(\cdot, \cdot)_{\mid t=t_{k}}$.

\subsection{A Galerkin Discretization}

We use a standard linear Lagrangian finite element to discretize the non-linear operators in the previous section. Finite element methods are particularly well suited to treat complex geometries, such as the one of the tokamak (plasma, passive structures, poloidal field coils.) We refer to section 5 for a general discussion on the choice of the order of the finite element method. For this we introduce a triangulation $\Omega_{h}$ of the domain $\Omega$ that resolves the subdomains $\Omega_{\mathrm{L}}, \Omega_{\mathrm{Fe}}, \Omega_{\mathrm{c}_{i, j}}, \Omega_{\mathrm{ps}_{k}}$. The finite element approximation $\psi_{h}$ of $\psi$ in the finite element space $V_{h}$ is an expansion in basis functions $\lambda_{i}$ :

$$
\psi_{h}(r, z)=\sum_{i} \psi_{i} \lambda_{i}(r, z) \text { with } \psi_{i} \in \mathbb{R} .
$$

Each Lagrangian basis function $\lambda_{i}(r, z)$ is piecewise linear and vanishes at all vertices except one. The domain of the plasma $\Omega_{\mathrm{p}}\left(\psi_{h}\right)$ of a finite element function $\psi_{h}$ is bounded by a continuous, polygonal, closed line. The critical points $\left(r_{\text {bnd }}\left(\psi_{h}\right), z_{\text {bnd }}\left(\psi_{h}\right)\right)$ and $\left(r_{\mathrm{ax}}\left(\psi_{h}\right), z_{\mathrm{ax}}\left(\psi_{h}\right)\right)$ are the coordinates of certain vertices of the mesh. The saddle point of a piecewise linear function $\psi_{h}$ is some vertex $\left(r_{0}, z_{0}\right)$ with the following property: if $\left(r_{1}, z_{1}\right),\left(r_{2}, z_{2}\right) \ldots\left(r_{n}, z_{n}\right)$, denote the counterclockwise ordered neighboring vertices the sequence of discrete gradients $\psi_{0}-\psi_{1}, \psi_{0}-\psi_{2} \ldots \psi_{0}-\psi_{n}$ changes at least four times the sign.

It remains to specify the quadrature rule that is used to approximate integrals over triangles $T$ and integrals over intersection $T \cap \Omega_{\mathrm{p}}\left(\psi_{h}\right)$ of triangles with the plasma domain

$$
\int_{T} f\left(r, \psi_{h}\right) \lambda_{i} d r d z \text { and } \int_{T \cap \Omega_{\mathrm{p}}\left(\psi_{h}\right)} g\left(r, \psi_{h}\right) \lambda_{i} d r d z .
$$

The second type of integrals appears in $J_{P}$ due to the fact that the mesh does not resolve the boundary of the plasma domain $\Omega_{\mathrm{p}}$. In any case we will use the centers of gravity

$$
\mathbf{b}_{T}:=\left(r_{T}, z_{T}\right) \quad \text { and } \quad \mathbf{b}_{T}\left(\psi_{h}\right):=\left(r_{T}\left(\psi_{h}\right), z_{T}\left(\psi_{h}\right)\right):=\left(r_{T \cap \Omega_{\mathrm{p}}\left(\psi_{h}\right)}, z_{T \cap \Omega_{\mathrm{p}}\left(\psi_{h}\right)}\right)
$$

of the integration domains $T$ or $T \cap \Omega_{\mathrm{p}}\left(\psi_{h}\right)$ as quadrature points. The corresponding quadrature weights are the size of the corresponding domain $|T|$ and $\left|T \cap \Omega_{\mathrm{p}}\left(\psi_{h}\right)\right|$. The barycenter for the second type of integrals depends itself on $\psi_{h}$. Our choice of quadrature rule introduces a consistency error of order $O\left(h^{2}\right)$, where $h$ is the diameter of the triangle, i.e. the quadrature is exact for linear integrands.

For a triangle $T$ with vertex coordinates $\mathbf{a}_{i}, \mathbf{a}_{j}, \mathbf{a}_{k} \in \mathbb{R}^{2}$ the center of gravity corresponds to the barycenter:

$$
\left(r_{T}, z_{T}\right)=\frac{1}{3}\left(\mathbf{a}_{i}+\mathbf{a}_{j}+\mathbf{a}_{k}\right)
$$



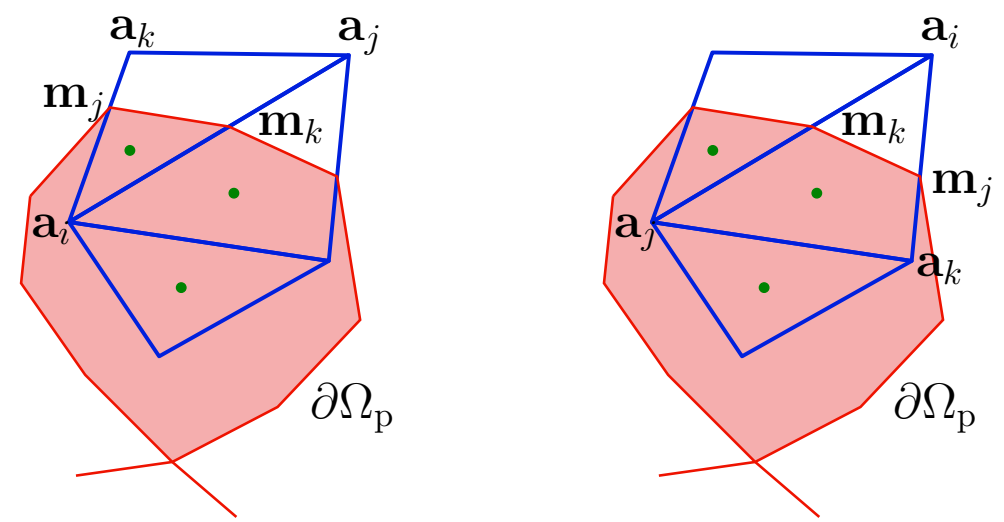

FiguRE 2. Integration over $T \cap \Omega_{\mathrm{p}}\left(\psi_{h}\right)$. The green dots indicate the location of the quadrature point. The integration domain $T \cap \Omega_{\mathrm{p}}\left(\psi_{h}\right)$ is either a) empty, b) the whole element $T$, c) a triangular domain or quadrilateral domain.

If the domain of integration is $T \cap \Omega_{\mathrm{p}}\left(\psi_{h}\right)$, we have to distinguish the two cases, where $T \cap \Omega_{\mathrm{p}}\left(\psi_{h}\right)$ is either a triangle or a quadrilateral. Without loss of generality we assume that $\mathbf{a}_{i}, \mathbf{a}_{j}, \mathbf{a}_{k}$ is a counterclockwise ordering of the vertex coordinates of $T$ and that $\partial \Omega_{\mathrm{p}}(\psi)$ intersects $\partial T$ at two points $\mathbf{m}_{k}$ and $\mathbf{m}_{j}$ at the edges opposite to the vertices $\mathbf{a}_{k}$ and $\mathbf{a}_{j}$ (See Figure 2). The barycentric coordinates of the intersecting points $\mathbf{m}_{k}$ and $\mathbf{m}_{j}$ are functions of $\psi_{h}$ :

$$
\lambda_{j}\left(\mathbf{m}_{k}\left(\psi_{h}\right)\right)=\frac{\psi_{\mathrm{bnd}}\left(\psi_{h}\right)-\psi_{i}}{\psi_{j}-\psi_{i}}, \quad \lambda_{k}\left(\mathbf{m}_{j}\left(\psi_{h}\right)\right)=\frac{\psi_{\mathrm{bnd}}\left(\psi_{h}\right)-\psi_{i}}{\psi_{k}-\psi_{i}},
$$

and, clearly, we have $\lambda_{k}\left(\mathbf{m}_{k}\left(\psi_{h}\right)\right)=\lambda_{j}\left(\mathbf{m}_{j}\left(\psi_{h}\right)\right)=0$.

If $T \cap \Omega_{\mathrm{p}}\left(\psi_{h}\right)$ is a triangle and $\mathbf{a}_{i}$ that vertex of $T$ that is contained in $T \cap \Omega_{\mathrm{p}}\left(\psi_{h}\right)$ (See Figure 2 left) we find:

$$
\left(r_{T}\left(\psi_{h}\right), z_{T}\left(\psi_{h}\right)\right)=\mathbf{a}_{i}+\frac{1}{3} \lambda_{j}\left(\mathbf{m}_{k}\left(\psi_{h}\right)\right)\left(\mathbf{a}_{j}-\mathbf{a}_{i}\right)+\frac{1}{3} \lambda_{k}\left(\mathbf{m}_{j}\left(\psi_{h}\right)\right)\left(\mathbf{a}_{k}-\mathbf{a}_{i}\right)
$$

and

$$
\left|T \cap \Omega_{\mathrm{p}}\left(\psi_{h}\right)\right|=|T| \lambda_{j}\left(\mathbf{m}_{k}\left(\psi_{h}\right)\right) \lambda_{k}\left(\mathbf{m}_{j}\left(\psi_{h}\right)\right) .
$$

If $T \cap \Omega_{\mathrm{p}}\left(\psi_{h}\right)$ is a quadrilateral and $\mathbf{a}_{i}$ that vertex of $T$ that is not contained in $T \cap \Omega_{\mathrm{p}}\left(\psi_{h}\right)$ (See Figure 2 right) we find:

$$
\begin{aligned}
&\left(r_{T}\left(\psi_{h}\right), z_{T}\left(\psi_{h}\right)\right)=\mathbf{a}_{i}+\frac{1}{3} \frac{1-\lambda_{j}^{2}\left(\mathbf{m}_{k}\left(\psi_{h}\right)\right) \lambda_{k}\left(\mathbf{m}_{j}\left(\psi_{h}\right)\right)}{1-\lambda_{j}\left(\mathbf{m}_{k}\left(\psi_{h}\right)\right) \lambda_{k}\left(\mathbf{m}_{j}\left(\psi_{h}\right)\right)}\left(\mathbf{a}_{j}-\mathbf{a}_{i}\right) \\
&+\frac{1}{3} \frac{1-\lambda_{j}\left(\mathbf{m}_{k}\left(\psi_{h}\right)\right) \lambda_{k}^{2}\left(\mathbf{m}_{j}\left(\psi_{h}\right)\right)}{1-\lambda_{j}\left(\mathbf{m}_{k}\left(\psi_{h}\right)\right) \lambda_{k}\left(\mathbf{m}_{j}\left(\psi_{h}\right)\right)}\left(\mathbf{a}_{k}-\mathbf{a}_{i}\right)
\end{aligned}
$$

and

$$
\left|T \cap \Omega_{\mathrm{p}}\left(\psi_{h}\right)\right|=|T|\left(1-\lambda_{j}\left(\mathbf{m}_{k}\left(\psi_{h}\right)\right) \lambda_{k}\left(\mathbf{m}_{j}\left(\psi_{h}\right)\right)\right) .
$$

In the next paragraph we will present a Newton method for the discretized non-linear problems, and it is important to work out accurately all the non-linear dependencies on the finite element solution $\psi_{h}$. Only then we can compute the correct derivatives.

For the sake of brevity we do not write down explicitly the discrete versions of the 
operators from the previous paragraph, but introduce the subcript $h$ to denote the discretized non-linear operators. $A_{h}$ for example is the discretized version of $A$. We get fully discrete non-linear formulations.

Galerkin Formulation 13 (Static). Let $S_{p^{\prime}}:[0,1] \rightarrow \mathbb{R}$ and $S_{f f^{\prime}}:[0,1] \rightarrow \mathbb{R}$ be two known functions and let the currents $I_{i}$ in the coils be given. We set $p^{\prime}(\psi)=S_{p^{\prime}}\left(\psi_{\mathrm{N}}\right)$ and $f f^{\prime}(\psi)=S_{f f^{\prime}}\left(\psi_{\mathrm{N}}\right)$ in $(3.2)$. We want to find $\psi_{h} \in V_{h}$ such that

$$
\mathrm{A}_{h}\left(\psi_{h}, \xi_{h}\right)-\mathrm{J}_{\mathrm{p}, h}\left(\psi_{h}, \xi_{h}\right)+\mathrm{c}_{h}\left(\psi_{h}, \xi_{h}\right)=\sum_{i=1}^{L} \sum_{j=1}^{N_{i}} \ell_{i, j, h}\left(I_{i, j}, \xi_{h}\right)
$$

holds for all $\xi_{h} \in V_{h}$.

Galerkin Formulation 14 (Static, with fixed plasma Current $I_{\mathrm{P}}$ ). Let $S_{p^{\prime}}$ : $[0,1] \rightarrow \mathbb{R}$ and $S_{f^{\prime}}:[0,1] \rightarrow \mathbb{R}$ be two known functions and let the currents $I_{i}$ in the coils be given. We set $p^{\prime}(\psi)=S_{p^{\prime}}\left(\psi_{\mathrm{N}}\right)$ and $f f^{\prime}(\psi)=S_{f f^{\prime}}\left(\psi_{\mathrm{N}}\right)$ in (3.2). Additionally we assume that the total plasma current $I_{\mathrm{P}}$ is given. We want to find $\psi_{h} \in V_{h}$ and $\lambda \in \mathbb{R}$ such that

$$
\begin{aligned}
\mathrm{A}_{h}\left(\psi_{h}, \xi_{h}\right)-\lambda \mathrm{J}_{\mathrm{p}, h}\left(\psi_{h}, \xi_{h}\right)+\mathrm{c}_{h}\left(\psi_{h}, \xi_{h}\right) & =\sum_{i=1}^{L} \sum_{j=1}^{N_{i}} \ell_{i, j, h}\left(I_{i, j}, \xi_{h}\right), \\
I_{\mathrm{p}}-\lambda \mathrm{J}_{\mathrm{p}, h}\left(\psi_{h}, 1\right) & =0,
\end{aligned}
$$

holds for all $\xi_{h} \in V_{h}$.

Galerkin Formulation 15 (Evolution). Let $S_{p^{\prime}}:[0,1] \times[0, T] \rightarrow \mathbb{R}$ and $S_{f f^{\prime}}$ : $[0,1] \times[0, T] \rightarrow \mathbb{R}$ be two known functions. Let the evolution of the voltages $\vec{V}_{i}(t)$ in the poloidal field circuits and the initial data $\psi_{0}$ be given. We set $p^{\prime}(\psi)=S_{p^{\prime}}\left(\psi_{\mathrm{N}}(t), t\right)$ and $f f^{\prime}(\psi)=S_{f f^{\prime}}\left(\psi_{\mathrm{N}}(t), t\right)$ in (3.2). We want to find $\psi_{h}^{k} \in V_{h}$ approximating $\psi\left(t_{k}\right)$ such that

$$
\begin{gathered}
\Delta t_{k} \mathrm{~A}_{h}\left(\psi_{h}^{k}, \xi_{h}\right)-\Delta t_{k} \mathrm{~J}_{\mathrm{p}, h}^{k}\left(\psi_{h}^{k}, \xi_{h}\right)-\mathrm{j}_{h}^{\mathrm{ps}}\left(\psi_{h}^{k}, \xi_{h}\right)-\mathrm{j}_{h}^{\mathrm{c}}\left(\psi_{h}^{k}, \xi_{h}\right)+\Delta t_{k} \mathrm{c}\left(\psi_{h}^{k}, \xi_{h}\right) \\
=\Delta t_{k} \sum_{i=1}^{L} \sum_{j=1}^{N_{i}} \ell_{i, j, h}\left(\left(\boldsymbol{S}_{i} \vec{V}_{i}\left(t_{k}\right)\right)_{j}, \xi_{h}\right)-\mathrm{j}_{h}^{\mathrm{ps}}\left(\psi_{h}^{k-1}, \xi_{h}\right)-\mathrm{j}_{h}^{\mathrm{c}}\left(\psi_{h}^{k-1}, \xi_{h}\right), \\
\psi_{h}^{0}=\psi_{0}
\end{gathered}
$$

holds for all $\xi_{h} \in V_{h}$ with $\mathrm{J}_{\mathrm{p}, h}^{k}(\cdot, \cdot)=\mathrm{J}_{\mathrm{p}, h}(\cdot, \cdot)_{\mid t=t_{k}}$.

Galerkin Formulation 16 (Evolution, with given plasma Current $I_{\mathrm{P}}(t)$ ). Let $S_{p^{\prime}}:[0,1] \times[0, T] \rightarrow \mathbb{R}$ and $S_{f f^{\prime}}:[0,1] \times[0, T] \rightarrow \mathbb{R}$ be two known functions. Let the evolution of the voltages $\vec{V}_{i}(t)$ in the poloidal field circuits and the initial data $\psi_{0}$ be given. We set $p^{\prime}(\psi)=S_{p^{\prime}}\left(\psi_{\mathrm{N}}(t), t\right)$ and $f f^{\prime}(\psi)=S_{f f^{\prime}}\left(\psi_{\mathrm{N}}(t), t\right)$ in (3.2). Additionally we assume that the evolution of the total plasma current $I_{\mathrm{P}}(t)$ is given. We want to find $\psi_{h}^{k} \in V_{h}$ and $\lambda^{k} \in \mathbb{R}$ approximating $\psi\left(t_{k}\right)$ and $\lambda\left(t_{k}\right)$ such that

$$
\begin{gathered}
\Delta t_{k} \mathrm{~A}_{h}\left(\psi_{h}^{k}, \xi_{h}\right)-\Delta t_{k} \mathrm{~J}_{\mathrm{p}, h}^{k}\left(\psi_{h}^{k}, \xi_{h}\right)-\mathrm{j}_{h}^{\mathrm{ps}}\left(\psi_{h}^{k}, \xi_{h}\right)-\mathrm{j}_{, h}^{\mathrm{c}}\left(\psi_{h}^{k}, \xi_{h}\right)+\Delta t_{k} \mathrm{c}\left(\psi_{h}^{k}, \xi_{h}\right) \\
=\Delta t_{k} \sum_{i=1}^{L} \sum_{j=1}^{N_{i}} \ell_{i, j, h}^{\mathrm{c}}\left(\left(\boldsymbol{S}_{i} \vec{V}_{i}\left(t_{k}\right)\right)_{j}, \xi_{h}\right)-\mathrm{j}_{h}^{\mathrm{ps}}\left(\psi_{h}^{k-1}, \xi_{h}\right)-\mathrm{j}_{, h}^{\mathrm{c}}\left(\psi_{h}^{k-1}, \xi_{h}\right), \\
I_{\mathrm{p}}\left(t_{k}\right)-\lambda^{k} \mathrm{~J}_{\mathrm{p}, h}^{k}\left(\psi_{h}^{k}, 1\right)=0, \quad \psi_{h}^{0}=\psi_{0}
\end{gathered}
$$

holds for all $\xi \in V_{h}$ with $\mathrm{J}_{\mathrm{p}, h}^{k}(\cdot, \cdot)=\mathrm{J}_{\mathrm{p}, h}(\cdot, \cdot)_{\mid t=t_{k}}$. 
The Galerkin formulations assume that the function $\mu_{\mathrm{Fe}}$ is known. In practical applications $\mu_{\mathrm{Fe}}$ needs to be estimated from experimental data. We refer to (Glowinski \& Marrocco 1974) and, more recently (Pechstein \& Jüttler 2006), for details.

\subsection{Newton's Method and the Free Plasma Boundary}

Newton's methods for solving a non-linear problem $F(x)=0$ for $x$ is the following iterative scheme:

$$
F^{\prime}\left(x_{i}\right)\left(x_{i+1}-x_{i}\right)=-F\left(x_{i}\right) \quad \Leftrightarrow \quad F^{\prime}\left(x_{i}\right) x_{i+1}=F^{\prime}\left(x_{i}\right) x_{i}-F\left(x_{i}\right) .
$$

If $F$ is sufficiently smooth, standard theory for Newton methods asserts that this iteration converges quadratically fast to the solution $x$. In our case the magnetic flux $\psi$ or its finite element approximation $\psi_{h}$ plays the role of the unknown $x$. If we want to apply this method to either our continuous non-linear variational formulations 9, 10, 11, and 12 or the discretized versions, namely the Galerkin Formulations 13, 14, 15, and 16, we need to compute derivatives of the non-linear operators.

For the continuous formulations we need to calculate all the directional derivatives $D_{\psi} \mathrm{A}(\psi, \xi)(\widetilde{\psi}), D_{\psi} \mathrm{J}_{\mathrm{p}}(\psi, \xi)(\widetilde{\psi}), D_{\psi} \mathrm{j}^{\mathrm{ps}}(\psi, \xi)(\tilde{\psi}), D_{\psi} \mathrm{j}^{\mathrm{c}}(\psi, \xi)(\tilde{\psi})$ and $D_{\psi} \mathrm{c}(\psi, \xi)(\tilde{\psi})$. This calculation is simple for the bilinear mappings $\mathrm{j}^{\mathrm{c}}$, $\mathrm{j}^{\mathrm{ps}}$, e.g.,

$$
D_{\psi \mathrm{j}^{\mathrm{ps}}}(\psi, \xi)(\tilde{\psi})=\mathrm{j}^{\mathrm{ps}}(\tilde{\psi}, \xi), \quad D_{\psi} \mathrm{j}^{\mathrm{c}}(\psi, \xi)(\tilde{\psi})=\mathrm{j}^{\mathrm{c}}(\tilde{\psi}, \xi),
$$

and the non-linear mapping $\mathrm{A}$ (see (2.6)):

$D_{\psi} \mathrm{A}(\psi, \xi)(\tilde{\psi})=\int_{\Omega} \frac{1}{\mu(\psi) r} \nabla \tilde{\psi} \cdot \nabla \xi d r d z-2 \int_{\Omega_{\mathrm{Fe}}} \frac{\mu_{\mathrm{Fe}}^{\prime}\left(|\operatorname{grad} \psi|^{2} r^{-2}\right)}{\mu_{\mathrm{Fe}}^{2}\left(|\operatorname{grad} \psi|^{2} r^{-2}\right) r^{3}} \nabla \tilde{\psi} \cdot \nabla \psi \nabla \psi \cdot \nabla \xi d r d z$.

The remaining derivative of $J_{p}$ was given in (Blum 1987, Lemma I.4):

$$
\begin{aligned}
D_{\psi} \mathrm{J}_{\mathrm{p}}(\psi, \xi)(\widetilde{\psi})= & \int_{\Omega_{\mathrm{p}}(\psi)} \frac{\partial j_{\mathrm{p}}\left(r, \psi_{\mathrm{N}}(\psi)\right)}{\partial \psi_{\mathrm{N}}} \frac{\partial \psi_{\mathrm{N}}(\psi)}{\partial \psi} \widetilde{\psi} \xi d r d z \\
& -\int_{\Gamma_{\mathrm{p}}(\psi)} j_{\mathrm{p}}(r, 1)|\nabla \psi|^{-1}\left(\widetilde{\psi}-\widetilde{\psi}\left(r_{\mathrm{bnd}}(\psi), z_{\mathrm{bnd}}(\psi)\right)\right) \xi d \Gamma \\
& +\int_{\Omega_{\mathrm{p}}(\psi)} \frac{\partial j_{\mathrm{p}}\left(r, \psi_{\mathrm{N}}(\psi)\right)}{\partial \psi_{\mathrm{N}}} \frac{\partial \psi_{\mathrm{N}}(\psi)}{\partial \psi_{\mathrm{ax}}} \widetilde{\psi}\left(r_{\mathrm{ax}}(\psi), z_{\mathrm{ax}}(\psi)\right) \xi d r d z, \\
& +\int_{\Omega_{\mathrm{p}}(\psi)} \frac{\partial j_{\mathrm{p}}\left(r, \psi_{\mathrm{N}}(\psi)\right)}{\partial \psi_{\mathrm{N}}} \frac{\partial \psi_{\mathrm{N}}(\psi)}{\partial \psi_{\mathrm{bnd}}} \widetilde{\psi}\left(r_{\mathrm{bnd}}(\psi), z_{\mathrm{bnd}}(\psi)\right) \xi d r d z,
\end{aligned}
$$

where $\Gamma_{\mathrm{p}}$ is the plasma boundary $\partial \Omega_{\mathrm{p}}$ and

$$
j_{\mathrm{p}}\left(r, \psi_{\mathrm{N}}(\psi)\right)=r S_{p^{\prime}}\left(\psi_{\mathrm{N}}(\psi)\right)+\frac{1}{\mu_{0} r} S_{f f^{\prime}}\left(\psi_{\mathrm{N}}(\psi)\right) .
$$

The derivation involves shape calculus (Murat \& Simon 1976, Delfour \& Zolésio 2011) and the non-trival derivatives:

$$
D_{\psi} \psi_{\mathrm{ax}}(\psi)(\tilde{\psi})=\tilde{\psi}\left(r_{\mathrm{ax}}(\psi), z_{\mathrm{ax}}(\psi)\right) \text { and } D_{\psi} \psi_{\mathrm{bnd}}(\psi)(\tilde{\psi})=\tilde{\psi}\left(r_{\mathrm{bnd}}(\psi), z_{\mathrm{bnd}}(\psi)\right) .
$$

The formula of the derivative relies on certain smoothness assumptions on $\psi$. Up to our knowledge, there is no theoretical evidence that this formula holds also for plasma equilibria with boundaries that contain $X$-points. In particular the second term on the right hand side seems to blow up if $\psi$ reaches a critical point.

Also in (Blum 1987), it is shown that the derivative of $J_{p}(\psi, \xi)$ in the direction $\psi$ 
vanishes: $D_{\psi} \mathrm{J}_{\mathrm{p}}(\psi, \xi)(\psi)=0$. Then the Newton scheme for solving Problem 10 is the following iteration: Let $\left(\psi^{n}, \lambda^{n}\right)$ be the solution at the $n$-th iteration. For given $\left(\psi^{n}, \lambda^{n}\right)$ we introduce the linear form:

$$
\begin{aligned}
F^{n}(\xi) & :=-\mathrm{A}\left(\psi^{n}, \xi\right)+D_{\psi} \mathrm{A}\left(\psi^{n}, \xi\right)\left(\psi^{n}\right)+\sum_{i=1}^{L} \sum_{j=1}^{N_{i}} \ell_{i, j}^{\mathrm{c}}\left(I_{i, j}, \xi\right) \\
& =-2 \int_{\Omega_{\mathrm{Fe}}} \frac{\mu_{\mathrm{Fe}}^{\prime}\left(|\nabla \psi|^{2} r^{-2}\right)}{\mu_{\mathrm{Fe}}^{2}\left(|\nabla \psi|^{2} r^{-2}\right) r^{3}}\left|\nabla \psi^{n}\right|^{2} \nabla \psi^{n} \cdot \nabla \xi d r d z+\sum_{i=1}^{L} \sum_{j=1}^{N_{i}} \ell_{i, j}^{\mathrm{c}}\left(I_{i, j}, \xi\right) .
\end{aligned}
$$

and the Newton update $\left(\psi^{n+1}, \lambda^{n+1}\right)$ is the solution of the infinite dimensional linear system

$$
\begin{aligned}
& D_{\psi} \mathrm{A}\left(\psi^{n}, \xi\right)\left(\psi^{n+1}\right)-\lambda^{n} D_{\psi} \mathrm{J}_{\mathrm{p}}\left(\psi^{n}, \xi\right)\left(\psi^{n+1}\right)+\mathrm{c}\left(\psi^{n+1}, \xi\right)-\mathrm{J}_{\mathrm{p}}\left(\psi^{n}, \xi\right) \lambda^{n+1}=F^{n}(\xi), \forall \xi \in V \\
& \lambda^{n} D_{\psi} \mathrm{J}_{\mathrm{p}}\left(\psi^{n}, 1\right)\left(\psi^{n+1}\right) \quad+\mathrm{J}_{\mathrm{p}}\left(\psi^{n}, 1\right) \lambda^{n+1}=I_{\mathrm{p}}
\end{aligned}
$$

After each iteration we need to recompute $\psi_{\mathrm{ax}}\left(\psi^{n}\right)=\psi^{n}\left(r_{\mathrm{ax}}\left(\psi^{n}\right), z_{\mathrm{ax}}\left(\psi^{n}\right)\right), \psi_{\text {bnd }}\left(\psi^{n}\right)=$ $\psi\left(r_{\text {bnd }}\left(\psi^{n}\right), z_{\text {bnd }}\left(\psi^{n}\right)\right)$ and $\Omega_{\mathrm{p}}\left(\psi^{n}\right)$. For the computation of the initial flux function $\psi^{0}$ we choose a constant permeability in iron and replace the non-linear form $\mathrm{J}_{\mathrm{p}}(\psi, \xi)$ with some linear form $\int_{\Omega_{\mathrm{p}}} j_{\text {init }} \xi d r d z$, where $\Omega_{\mathrm{p}}$ is a given ellipse and $j_{\text {init }}$ a given constant current density. Hence $\psi^{0}$ is the solution to a linear problem and determines the plasma axis and the plasma boundary in the first Newton iteration.

The Newton iterations for the Problems 9, 11 and 12 follow likewise. The equilibrium codes SCED (Blum et al. 1981) and Proteus (Albanese et al. 1987) are based on discretizations of such Newton iterations. The flux functions $\psi^{n}$ and $\psi^{n+1}$ are approximated by finite weighted sums of finite element basis functions and the test functions $\xi$ cycle over all test functions. In each Newton iteration, one has to invert an algebraic system whose size is equal to the number of finite element basis functions. But since it is not clear, whether the formula for the derivative of $\mathrm{J}_{\mathrm{p}}(\psi, \xi)(\widetilde{\psi})$ remains valid for plasma boundaries with X-points, these approaches are not very trustworthy.

In CEDRES++ we prefer to use Newton methods for the Galerkin Formulations 13 , 14 and 16 . Such Newton methods need the directional derivatives $D_{\psi_{h}} \mathrm{~A}_{h}\left(\psi_{h}, \xi_{h}\right)\left(\bar{\psi}_{h}\right)$, $D_{\psi_{h}} \mathrm{~J}_{\mathrm{p}, h}\left(\psi_{h}, \xi_{h}\right)\left(\widetilde{\psi}_{h}\right), D_{\psi_{h}} \mathrm{j}_{h}^{\mathrm{ps}}\left(\psi_{h}, \xi_{h}\right)\left(\tilde{\psi}_{h}\right), D_{\psi_{h}} \mathrm{j}_{h}^{\mathrm{c}}\left(\psi_{h}, \xi_{h}\right)\left(\tilde{\psi}_{h}\right)$ and $D_{\psi_{h}} \mathrm{c}_{h}\left(\psi_{h}, \xi_{h}\right)\left(\tilde{\psi}_{h}\right)$. Here again, this is a straightforward and simple calculation for all mappings except one: the mapping $J_{\mathrm{P}, h}$ that is related to the non-linear current profile in the plasma domain. The mapping $\mathbf{J}_{\mathrm{P}, h}$ is given by

$$
\mathrm{J}_{\mathrm{P}, h}\left(\psi_{h}, \lambda_{m}\right)=\sum_{T} \mathrm{~J}_{\mathrm{P}, h}^{T}\left(\psi_{h}, \lambda_{m}\right):=\sum_{T}\left|T \cap \Omega_{\mathrm{p}}\left(\psi_{h}\right)\right| j_{\mathrm{p}}\left(\mathbf{b}_{T}\left(\psi_{h}\right)\right) \lambda_{m}\left(\mathbf{b}_{T}\left(\psi_{h}\right)\right),
$$

where $j_{\mathrm{p}}\left(\mathbf{b}_{T}\left(\psi_{h}\right)\right)=j_{\mathrm{p}}\left(r_{T}\left(\psi_{h}\right), \psi_{\mathrm{N}}\left(\psi_{h}\left(\mathbf{b}_{T}\left(\psi_{h}\right)\right), \psi_{\mathrm{ax}}\left(\psi_{h}\right), \psi_{\text {bnd }}\left(\psi_{h}\right)\right)\right)$. The directional derivative of $\mathrm{J}_{\mathrm{P}, h}\left(\psi_{h}, \lambda_{m}\right)$ in direction $\lambda_{n}$ is the partial derivative with respect to the expansion coefficient $\psi_{n}$ :

$$
D_{\psi} \mathrm{J}_{\mathrm{P}, h}\left(\psi_{h}, \lambda_{m}\right)\left(\lambda_{n}\right)=\frac{\partial}{\partial \psi_{n}} \mathrm{~J}_{\mathrm{P}, h}\left(\psi_{h}, \lambda_{m}\right)=\frac{\partial}{\partial \psi_{n}} \mathrm{~J}_{\mathrm{P}, h}\left(\sum_{i} \psi_{i} \lambda_{i}, \lambda_{m}\right)
$$

Computing the derivative of each terms of $\mathrm{J}_{\mathrm{P}, h}\left(\psi_{h}, \lambda_{m}\right)$ is a tedious application of chain and product rules. We distinguish three different cases: $T \cap \Omega_{\mathrm{p}}\left(\psi_{h}\right)=0, T \cap \Omega_{\mathrm{p}}\left(\psi_{h}\right)=T$ and $T \cap \Omega_{\mathrm{p}}\left(\psi_{h}\right) \subset T$ (see Figure 2). With a slight abuse of notation we identify $\psi_{\text {bnd }}$ and $\psi_{\text {ax }}$ with the corresponding finite element expansion coefficient and use the Kronecker deltas $\delta_{n, \text { bnd }}$ and $\delta_{n, \text { ax }}$. 
(a) $T \cap \Omega_{\mathrm{p}}\left(\psi_{h}\right)=0$ :

$$
\frac{\partial}{\partial \psi_{n}} J_{\mathrm{P}, h}^{T}\left(\psi_{h}, \lambda_{m}\right)=0
$$

(b) $T \cap \Omega_{\mathrm{p}}\left(\psi_{h}\right)=T$ :

$$
\begin{aligned}
\frac{\partial}{\partial \psi_{n}} \mathbf{J}_{\mathrm{P}, h}^{T}\left(\psi_{h}, \lambda_{m}\right)=|T| \frac{\partial j_{\mathrm{p}}\left(r_{T}, \psi_{\mathrm{N}}\left(\mathbf{b}_{T}\right)\right)}{\partial \psi_{\mathrm{N}}}\left(\frac{\partial \psi_{\mathrm{N}}\left(\mathbf{b}_{T}\right)}{\partial \psi_{h}} \lambda_{n}\left(\mathbf{b}_{T}\right)\right. \\
\left.+\frac{\partial \psi_{\mathrm{N}}\left(\mathbf{b}_{T}\right)}{\partial \psi_{\text {bnd }}} \delta_{n, \text { bnd }}+\frac{\partial \psi_{\mathrm{N}}\left(\mathbf{b}_{T}\right)}{\partial \psi_{\mathrm{ax}}} \delta_{n, \text { ax }}\right) \lambda_{m}\left(\mathbf{b}_{T}\right)
\end{aligned}
$$

(c) $T \cap \Omega_{\mathrm{p}}\left(\psi_{h}\right) \subset T$ : Without loss of generality we adopt the notation from section 3.2 introduce $\lambda_{j}^{k}=\lambda_{j}\left(\mathbf{m}_{k}\right)$ and $\lambda_{k}^{j}=\lambda_{k}\left(\mathbf{m}_{j}\right)$ use $\mathbf{b}_{T}$ to denote $\mathbf{b}_{T}\left(\psi_{h}\right)$. We define $A R=|T| \lambda_{j}^{k} \lambda_{k}^{j}$ if $T \cap \Omega_{\mathrm{p}}\left(\psi_{h}\right)$ is a triangle and $A R=|T|\left(1-\lambda_{j}^{k} \lambda_{k}^{j}\right)$ if $T \cap \Omega_{\mathrm{p}}\left(\psi_{h}\right)$ is a quadrilateral. We find

$$
\frac{\partial}{\partial \psi_{n}} \mathrm{~J}_{\mathrm{P}, h}^{T}\left(\psi_{h}, \lambda_{m}\right)=A_{n}^{T}\left(\psi_{h}, \lambda_{m}\right)+C_{n}^{T}\left(\psi_{h}, \lambda_{m}\right)+T_{n}^{T}\left(\psi_{h}, \lambda_{m}\right)
$$

with

- the derivative related to the area $\left|T \cap \Omega_{\mathrm{p}}\left(\psi_{h}\right)\right|$ :

$$
\begin{aligned}
A_{n}^{T}\left(\psi_{h}, \lambda_{m}\right)=s|T|\left(\left(\frac{\partial \lambda_{j}^{k}}{\partial \psi_{n}} \lambda_{k}^{j}+\lambda_{k}^{j} \frac{\partial \lambda_{j}^{k}}{\partial \psi_{n}}\right)\right. & \\
& \left.+\left(\frac{\partial \lambda_{j}^{k}}{\partial \psi_{\mathrm{bnd}}} \lambda_{k}^{j}+\lambda_{k}^{j} \frac{\partial \lambda_{j}^{k}}{\partial \psi_{\mathrm{bnd}}}\right) \delta_{n, \text { bnd }}\right) j_{\mathrm{p}}\left(\mathbf{b}_{T}\right) \lambda_{m}\left(\mathbf{b}_{T}\right),
\end{aligned}
$$

where $s=1$ if $\left|T \cap \Omega_{\mathrm{p}}\left(\psi_{h}\right)\right|$ is a triangle and $s=-1$ else.

- the derivative related to the current $j_{\mathrm{p}}\left(r_{T}, \psi_{\mathrm{N}}\left(\mathbf{b}_{T}\right)\right)$ :

$$
\begin{aligned}
C_{n}^{T}\left(\psi_{h}, \lambda_{m}\right)= & A R \frac{\partial j_{\mathrm{p}}\left(r_{T}, \psi_{\mathrm{N}}\left(\mathbf{b}_{T}\right)\right)}{\partial r}\left(\frac{\partial r_{T}}{\partial \psi_{n}} \lambda_{n}\left(\mathbf{b}_{T}\right)+\frac{\partial r_{T}}{\partial \psi_{\mathrm{bnd}}} \delta_{n, \text { bnd }}\right) \lambda_{m}\left(\mathbf{b}_{T}\right) \\
& +A R \frac{\partial j_{\mathrm{p}}\left(r_{T}, \psi_{\mathrm{N}}\left(\mathbf{b}_{T}\right)\right)}{\partial \psi_{\mathrm{N}}}\left(\frac{\partial \psi_{\mathrm{N}}\left(\mathbf{b}_{T}\right)}{\partial \psi_{\mathrm{bnd}}} \delta_{n, \text { bnd }}+\frac{\partial \psi_{\mathrm{N}}\left(\mathbf{b}_{T}\right)}{\partial \psi_{\mathrm{ax}}} \delta_{n, \mathrm{ax}}\right) \lambda_{m}\left(\mathbf{b}_{T}\right) \\
& +A R \frac{\partial j_{\mathrm{p}}\left(r_{T}, \psi_{\mathrm{N}}\left(\mathbf{b}_{T}\right)\right)}{\partial \psi_{\mathrm{N}}}\left(\frac{\partial \psi_{\mathrm{N}}\left(\mathbf{b}_{T}\right)}{\partial \psi_{h}} \lambda_{n}\left(\mathbf{b}_{T}\right)+\frac{\partial \psi_{\mathrm{N}}\left(\mathbf{b}_{T}\right)}{\partial \psi_{h}} \nabla \psi_{h}\left(\mathbf{b}_{T}\right) \cdot \frac{\partial \mathbf{b}_{T}}{\partial \psi_{n}}\right. \\
& \left.+\frac{\partial \psi_{\mathrm{N}}\left(\mathbf{b}_{T}\right)}{\partial \psi_{h}} \nabla \psi_{h}\left(\mathbf{b}_{T}\right) \cdot \frac{\partial \mathbf{b}_{T}}{\partial \psi_{\mathrm{bnd}}} \delta_{n, \text { bnd }}\right) \lambda_{m}\left(\mathbf{b}_{T}\right)
\end{aligned}
$$

- the derivative related to the test function $\lambda_{m}\left(\mathbf{b}_{T}\right)$ :

$$
T_{n}^{T}\left(\psi_{h}, \lambda_{m}\right)=A R j_{\mathrm{p}}\left(\mathbf{b}_{T}\right)\left(\nabla \lambda_{m}\left(\mathbf{b}_{T}\right) \cdot \frac{\partial \mathbf{b}_{T}}{\partial \psi_{n}}+\nabla \lambda_{m}\left(\mathbf{b}_{T}\right) \cdot \frac{\partial \mathbf{b}_{T}}{\partial \psi_{\text {bnd }}} \delta_{n, \text { bnd }}\right)
$$

The derivatives of $\psi_{\mathrm{N}}$ follow easily from the definition (2.9). We would like to stress that the Galerkin matrix $D_{\psi} \mathrm{J}_{\mathrm{P}, h}^{T}\left(\psi_{h}, \lambda_{m}\right)\left(\lambda_{n}\right)$ can be assembled in a fairly standard, i.e. element wise, fashion, provided we compute in a preprocessing step the following information for each element element $T$ belonging to the last case: We need to know the barycententric coordinates of the intersection points $\lambda_{k}\left(\mathbf{m}_{j}\right)$ and $\lambda_{j}\left(\mathbf{m}_{k}\right)$, the barycenter $\mathbf{b}_{T}\left(\psi_{h}\right)$ and the derivatives $\frac{\partial \lambda_{k}\left(\mathbf{m}_{j}\right)}{\partial \psi_{i}}, \frac{\partial \lambda_{k}\left(\mathbf{m}_{j}\right)}{\partial \psi_{j}}, \frac{\partial \lambda_{k}\left(\mathbf{m}_{j}\right)}{\partial \psi_{k}}, \frac{\partial \lambda_{k}\left(\mathbf{m}_{j}\right)}{\partial \psi_{\text {bnd }}}, \frac{\partial \lambda_{j}\left(\mathbf{m}_{k}\right)}{\partial \psi_{i}}, \frac{\partial \lambda_{j}\left(\mathbf{m}_{k}\right)}{\partial \psi_{j}}$, 
$\frac{\partial \lambda_{j}\left(\mathbf{m}_{k}\right)}{\partial \psi_{k}}, \frac{\partial \lambda_{j}\left(\mathbf{m}_{k}\right)}{\partial \psi_{\text {bnd }}}$ and $\frac{\partial \mathbf{b}_{T}}{\partial \psi_{i}}, \frac{\partial \mathbf{b}_{T}}{\partial \psi_{j}}, \frac{\partial \mathbf{b}_{T}}{\partial \psi_{k}}, \frac{\partial \mathbf{b}_{T}}{\partial \psi_{\text {bnd }}}$. All this information can be easily computed for given $\psi_{h}, \psi_{\text {bnd }}$ and $\psi_{\text {ax }}$ using the formulas (3.14), (3.15) and (3.17). All the terms that contain the Kronecker deltas $\delta_{n, \text { bnd }}$ or $\delta_{n \text {,ax }}$ lead to non-local entries in the stiffness matrix. They connect the coefficients $\psi_{i_{1}}=\psi_{\text {bnd }}$ and $\psi_{i_{2}}=\psi_{\text {ax }}$ with all coefficients $\psi_{j}$ that are associated to vertices of elements that are intersected by the plasma domain $\Omega_{\mathrm{P}}\left(\psi_{h}\right)$.

The size of the algebraic systems that we need to solve in each iteration corresponds to the number of vertices of the triangulation. Even for very fine discretizations it is today possible to use direct linear solvers such as UMFPACK (Davis 2011). As long as the storage amount for the algebraic system does not exceed the memory, modern direct solvers will outperform in most cases an iterative solver.

\subsection{Sequential Quadratic Programming for the Inverse Problems}

In CEDRES++ we use the following fully discrete reformulation of the inverse Problems 3 and 4 to find optimal currents in the poloidal field coils.

Inverse Problem 17 (Static). Let $S_{p^{\prime}}:[0,1] \rightarrow \mathbb{R}$ and $S_{f f^{\prime}}:[0,1] \rightarrow \mathbb{R}$ be two known functions. We set $p^{\prime}(\psi)=S_{p^{\prime}}\left(\psi_{\mathrm{N}}\right)$ and $f f^{\prime}(\psi)=S_{f f^{\prime}}\left(\psi_{\mathrm{N}}\right)$ in 3.2 . We solve the following minimization problem:

$$
\min _{\psi_{h}, I_{1,1}, \ldots I_{L, N_{L}}} K\left(\psi_{h}\right)+R\left(I_{1,1}, \ldots I_{L, N_{L}}\right) \quad \text { subject to } 3.19 \text {. }
$$

Inverse Problem 18 (Static, with given Plasma Current $I_{\mathrm{P}}$ ). Let $S_{p^{\prime}}:[0,1] \rightarrow$ $\mathbb{R}$ and $S_{f f^{\prime}}:[0,1] \rightarrow \mathbb{R}$ be two known functions and assume additionally that the total plasma current $I_{\mathrm{P}}$ is given. We set $p^{\prime}(\psi)=S_{p^{\prime}}\left(\psi_{\mathrm{N}}\right)$ and $f f^{\prime}(\psi)=S_{f f^{\prime}}\left(\psi_{\mathrm{N}}\right)$ in 3.2 . We solve the following minimization problem:

$$
\min _{\lambda, \psi_{h}, I_{1,1}, \ldots I_{L, N_{L}}} K\left(\psi_{h}\right)+R\left(I_{1,1}, \ldots I_{L, N_{L}}\right) \quad \text { subject to } 3.20 \text {. }
$$

The inverse Problems 17 and 18 are finite dimensional constrained optimization problems. The sequential quadratic programming (SQP) method is the fastest method for finite dimensional constrained optimization problems. We refer to the text book (Nocedal \& Wright 2006. Chapter 18) for the details and explain here only the basic idea.

Both inverse Problems 17 and 18 are optimization problems of the following type

$$
\min _{\mathbf{u}, \mathbf{y}} \frac{1}{2} \mathbf{y}^{T} \boldsymbol{K} \mathbf{y}+\frac{1}{2} \mathbf{u}^{T} \boldsymbol{H} \mathbf{u} \quad \text { s.t } \quad \boldsymbol{B}(\mathbf{y})=\boldsymbol{F}(\mathbf{u}),
$$

where the quadratic matrices $\boldsymbol{H}$ and $\boldsymbol{K}$ are the discretization of the cost functions $K$ and $R$, the state variable $\mathbf{y}$ is the vector of the finite element coefficients $\psi_{i}$ and the scaling factor $\lambda$, the control variable $\mathbf{u}$ is the vector of the $N$ currents $I_{i}$ in the poloidal field coils and $\boldsymbol{B}$ and $\boldsymbol{F}$ the Galerkin discretizations of (3.19) or (3.20). The Lagrange function formalism in combination with Newton-type iterations is one approach to derive the SQP-methods: the Lagrangian for 3.30 is

$$
L(\mathbf{y}, \mathbf{u}, \mathbf{p})=\frac{1}{2} \mathbf{y}^{T} \boldsymbol{K} \mathbf{y}+\frac{1}{2} \mathbf{u}^{T} \boldsymbol{H} \mathbf{u}+\mathbf{p}^{T}(\boldsymbol{B}(\mathbf{y})-\boldsymbol{F}(\mathbf{u}))
$$


and the solution of 3.30 is a stationary point of this Lagrangian:

$$
\begin{aligned}
\boldsymbol{K} \mathbf{y}+D_{\mathbf{y}} \boldsymbol{B}^{T}(\mathbf{y}) \mathbf{p} & =0 \\
\boldsymbol{H u}-D_{\mathbf{u}} \boldsymbol{F}^{T}(\mathbf{u}) \mathbf{p} & =0 \\
\boldsymbol{B}(\mathbf{y})-\boldsymbol{F}(\mathbf{u}) & =0
\end{aligned}
$$

The superscript $T$ indicates the adjoint operator, which corresponds to matrix transposition in the finite dimensional case. The second line in $(3.32)$ corresponds to the optimality condition for the gradient of the reduced cost functional $\frac{1}{2} \mathbf{u}^{T} \boldsymbol{H} \mathbf{u}+\frac{1}{2} \mathbf{y}^{T}(\mathbf{u}) \boldsymbol{K y}(\mathbf{u})$, where $\mathbf{y}(\mathbf{u})$ is implicitly defined by $\boldsymbol{B}(\mathbf{y}(\mathbf{u}))=\boldsymbol{F}(\mathbf{u})$. This is the main reason for which gradient type methods for a corresponding unconstrained optimization problem for the reduced cost function are too expensive: one evaluation of the gradient requires the very expensive solution of the non-linear problem in the third line of $(3.32)$. For the SQP-methods on the other hand, the overall computing time in practical examples has about the same magnitude as the computing time for solving the constraint for given control parameters.

A quasi-Newton method for solving (3.32) are iterations of the type

$$
\left(\begin{array}{ccc}
\boldsymbol{K} & 0 & D_{\mathbf{y}} \boldsymbol{B}^{T}\left(\mathbf{y}^{i}\right) \\
0 & \boldsymbol{H} & -D_{\mathbf{u}} \boldsymbol{F}^{T}\left(\mathbf{u}^{i}\right) \\
D_{\mathbf{y}} \boldsymbol{B}\left(\mathbf{y}^{i}\right)-D_{\mathbf{u}} \boldsymbol{F}\left(\mathbf{u}^{i}\right) & 0
\end{array}\right)\left(\begin{array}{c}
\mathbf{y}^{i+1}-\mathbf{y}^{i} \\
\mathbf{u}^{i+1}-\mathbf{u}^{i} \\
\mathbf{p}^{i+1}-\mathbf{p}^{i}
\end{array}\right)=-\left(\begin{array}{c}
\boldsymbol{K} \mathbf{y}^{i}+D_{\mathbf{y}} \boldsymbol{B}^{T}\left(\mathbf{y}^{i}\right) \mathbf{p}^{i} \\
\boldsymbol{H} \mathbf{u}^{i}-D_{\mathbf{u}} \boldsymbol{F}^{T}\left(\mathbf{u}^{i}\right) \mathbf{p}^{i} \\
\boldsymbol{B}\left(\mathbf{y}^{i}\right)-\boldsymbol{F}\left(\mathbf{u}^{i}\right)
\end{array}\right)
$$

We call the iterative scheme 3.33 a quasi-Newton method since we omit the second order derivatives of $\boldsymbol{B}$ and $\boldsymbol{F}$. The quadratic convergence of Newton's method deteriorates to super-linear convergence. The number of control parameters is much smaller than the number of state coefficients. Therefore the algebraic system in (3.33) is roughly twice as large as the algebraic system of a Newton iteration of the direct problem. Hence, as in the direct case, there is today no need to use iterative linear solver.

This will be different for the inverse problems of evolving free-boundary equilibria. There the size of the algebraic system increases by a factor that corresponds to the number of timesteps. We refer for (Blum \& Heumann 2014) for details and state here only the finite dimensional inverse problems that are addressed in CEDRES ++ . They are based on a discrete cost function $K_{h}\left(\left\{\psi_{h}^{k}\right\}_{k=1}^{n}\right)$ :

$$
K_{h}\left(\left\{\psi_{h}^{k}\right\}_{k=1}^{n}\right)=\sum_{k=1}^{n}\left(\frac{\Delta t_{k}}{2} \sum_{i=1}^{N_{\text {desi }}}\left(\psi_{h}^{k}\left(r_{i}, z_{i}\right)-\psi_{h}^{k}\left(r_{\text {desi }}\left(t_{k}\right), z_{\text {desi }}\left(t_{k}\right)\right)\right)^{2}\right)
$$

for the finite element approximation $\left\{\psi_{h}^{k}\right\}_{k=1}^{n}$ at $t_{k}$ and a discrete regularization function:

$$
R_{h}\left(\left\{\vec{V}_{1}\left(t_{k}\right)\right\}_{k=1}^{n}, \ldots,\left\{\vec{V}_{L}\left(t_{k}\right)\right\}_{k=1}^{n}\right)=\sum_{i=1}^{L} \frac{w_{i}}{2} \sum_{k=1}^{n} \Delta t_{k} \vec{V}_{i}\left(t_{k}\right) \cdot \vec{V}_{i}\left(t_{k}\right) .
$$

for the coil voltages $\left\{\vec{V}_{i}\left(t_{k}\right)\right\}_{k=1}^{n}$.

Inverse Problem 19 (Evolution). Let $S_{p^{\prime}}:[0,1] \times \mathbb{R} \rightarrow \mathbb{R}$ and $S_{f f^{\prime}}:[0,1] \times \mathbb{R} \rightarrow \mathbb{R}$ be two known functions. We set $p^{\prime}(\psi)=S_{p^{\prime}}\left(\psi_{\mathrm{N}}(t), t\right)$ and $f f^{\prime}(\psi)=S_{f f^{\prime}}\left(\psi_{\mathrm{N}}(t), t\right)$ in (3.2). We solve the following minimization problem:

$$
\min _{\left\{\psi_{h}^{k}, \vec{V}_{i}\left(t_{k}\right)\right\}_{k=1}^{n}} K_{h}\left(\left\{\psi_{h}^{k}\right\}_{k=1}^{n}\right)+R_{h}\left(\left\{\vec{V}_{1}\left(t_{k}\right)\right\}_{k=1}^{n}, \ldots,\left\{\vec{V}_{L}\left(t_{k}\right)\right\}_{k=1}^{n}\right) \quad \text { subject to 3.21]. }
$$

Inverse Problem 20 (Evolution, with given plasma Current $I_{\mathrm{P}}$ ). Let $S_{p^{\prime}}$ : $[0,1] \times \mathbb{R} \rightarrow \mathbb{R}$ and $S_{f^{\prime}}:[0,1] \times \mathbb{R} \rightarrow \mathbb{R}$ be two known functions and assume additionally 
that the total plasma current $I_{\mathrm{P}}$ is given. We set $p^{\prime}(\psi)=S_{p^{\prime}}\left(\psi_{\mathrm{N}}(t), t\right)$ and $f f^{\prime}(\psi)=$ $S_{f f^{\prime}}\left(\psi_{\mathrm{N}}(t), t\right)$ in 3.2 . We solve the following minimization problem:

$$
\min _{\left\{\lambda^{k}, \psi_{h}^{k}, \vec{V}_{i}\left(t_{k}\right)\right\}_{k=1}^{n}} K_{h}\left(\left\{\psi_{h}^{k}\right\}_{k=1}^{n}\right)+R_{h}\left(\left\{\vec{V}_{1}\left(t_{k}\right)\right\}_{k=1}^{n}, \ldots,\left\{\vec{V}_{L}\left(t_{k}\right)\right\}_{k=1}^{n}\right) \quad \text { subject to 3.22. }
$$

We would like to highlight that the SQP-method relies on proper derivatives of the nonlinear operators $\boldsymbol{B}$ and $\boldsymbol{F}$. In our case $\boldsymbol{F}$ is affine, hence the derivative of $\boldsymbol{B}$ remains the most difficult part. On the other hand these derivatives are exactly the same derivatives that we used for the new Newton methods. Hence the implementation of a SQP-method for the inverse problem uses the same main building blocks.

\subsection{Flux Surface Averages and Geometric Coefficients}

As for any equilibrium code numerous outputs can be extracted from the poloidal flux map computed. These include purely geometric information on the plasma shape (plasma boundary, geometric axis, elongation ...), global parameters (such as total plasma current $I_{\mathrm{P}}$, poloidal beta $\beta_{p}$, internal inductance $\left.l i, \ldots\right), 1 \mathrm{D}$ profiles of quantities constant on flux isolines in the plasma and 2D maps ( $\psi$ itself but also $\left.B_{r}, B_{z}, j_{p}, \ldots\right)$. All these outputs are standardized and follow the conventions of the European Integrated Tokamak Modelling Project (Falchetto et al. 2014, ITM 2013). We are not going to detail all of them in this paper. Let us however give some details on the computation of some of the important 1D profiles in the plasma. For $\psi_{\mathrm{N}} \in[0,1], S_{f}\left(\psi_{\mathrm{N}}\right)=f(\psi)$ is computed by integration of $S_{f f^{\prime}}$

$$
S_{f}\left(\psi_{\mathrm{N}}\right)=\left[\left(r_{0} B_{0}\right)^{2}-2\left(\psi_{\mathrm{bnd}}-\psi_{\mathrm{ax}}\right) \int_{\psi_{\mathrm{N}}}^{1} S_{f f^{\prime}}(x) d x\right]^{1 / 2},
$$

where $B_{0}$ is the vacuum toroidal field at $r=r_{0}$. Let us define a discretization of the unit interval $[0,1]$ by $S+1$ values $\psi_{\mathrm{N}}^{0}=0, \ldots, \psi_{\mathrm{N}}^{S}=1$. These points are taken as abscissa for all computed 1D profiles. For each $\psi_{\mathrm{N}}^{s}$ the contour line $\Gamma_{\psi_{\mathrm{N}}^{s}}$ is extracted from the finite element representation of the solution as a list of $N_{s}$ segments between $\mathbf{m}_{s, 1}^{l}=\left(r_{s, 1}^{l}, z_{s, 1}^{l}\right)$ and $\mathbf{m}_{s, 2}^{l}=\left(r_{s, 2}^{l}, z_{s, 2}^{l}\right)$ with length $\left|L_{s}^{l}\right|$, for $l=1$ to $N_{s}$.

The toroidal flux coordinate is defined as $\rho\left(\psi_{\mathrm{N}}\right)=\sqrt{\phi\left(\psi_{\mathrm{N}}\right) / \pi B_{0}}$ where $\phi\left(\psi_{\mathrm{N}}\right)=$ $\int_{\Omega_{\psi_{N}}} \frac{f(\psi(r, z))}{r} d r d z$ and $\Omega_{\psi_{N}}$ is the domain bounded by the line of flux $\Gamma_{\psi_{\mathrm{N}}}$. The quantities $\phi_{s}$ and $\rho_{s}$ are computed from the discrete $\psi_{h}$ for all $\psi_{\mathrm{N}}^{s}$ using a barycentric quadrature rule (cf. Section 3.2):

$$
\phi_{s}=\sum_{T} \frac{S_{f}\left(\psi_{\mathrm{N}}\left(\mathbf{b}_{T}\left(\psi_{h}\right)\right)\right)}{r_{T}\left(\psi_{h}\right)}\left|T \cap \Omega_{\psi_{\mathrm{N}}^{s}}\right| .
$$

The profiles $\psi_{s}$ and $\rho_{s}$ being known one can compute $\left(\frac{\partial \psi}{\partial \rho}\right)_{s}=\psi_{s}^{\prime}$ using finite differences.

In the same way the volume profile is computed as

$$
\operatorname{Vol}_{s}=2 \pi \sum_{T} r_{T}\left(\psi_{h}\right)\left|T \cap \Omega_{\psi_{\mathrm{N}}^{s}}\right|
$$

and $\left(\frac{\partial V o l}{\partial \rho}\right)_{s}=V o l_{s}^{\prime}$ using finite differences.

Following (Blum 1987) the average of a quantity $A$ over magnetic surfaces can be computed as

$$
\langle A\rangle_{s}=\left(\int_{\Gamma_{\psi_{\mathrm{N}}^{s}}} \frac{A r}{\left|\nabla \psi_{h}\right|} d l\right) /\left(\int_{\Gamma_{\psi_{\mathrm{N}}^{\mathrm{s}}}} \frac{r}{\left|\nabla \psi_{h}\right|} d l\right) .
$$


A number of 1D profiles, also called geometric coefficients, are computed as such averages, e.g. $\left\langle 1 / r^{2}\right\rangle$ or $\left\langle|\nabla \rho|^{2} / r^{2}\right\rangle$. The integrals over flux contour lines involved are approximated as follows:

$$
\int_{\Gamma_{\psi_{\mathrm{N}}^{s}}} \frac{A r}{\left|\nabla \psi_{h}\right|} d l \approx \sum_{l=1}^{N_{s}} \frac{1}{2}\left(\frac{r_{s, 1}^{l} A\left(\mathbf{m}_{s, 1}^{l}\right)}{\left|\nabla \psi_{h \mid T_{s}^{l}}\right|}+\frac{r_{s, 2}^{l} A\left(\mathbf{m}_{s, 2}^{l}\right)}{\left|\nabla \psi_{h \mid T_{s}^{l}}\right|}\right)\left|L_{s}^{l}\right| .
$$

where $T_{s}^{l}$ is the triangle which is intersected by the segment between $\mathbf{m}_{s, 1}^{l}$ and $\mathbf{m}_{s, 2}^{l}$ and $\mathbf{m}_{s, \cdot}^{l}=\left(r_{s, \cdot}^{l}, z_{s,}^{l}\right) \cdot\left|\nabla \psi_{h \mid T_{s}^{l}}\right|$ is constant in the triangle and computed from the 3 values at the nodes of $T_{s}^{l}$.

\subsection{Volume integrated Poynting theorems}

The subset of equations (2.1) and (2.2) we used in section 2 to derive the evolution problems 5 and 6 that are solved in CEDRES ++ involve the poloidal Faraday and the toroidal Ampère law. Hence, the poloidal Poynting theorem 2.4 can be used to check the accuracy of the solution independently of an additional treatment of the transport equations.

We integrate the poloidal Poynting theorem (2.4) over a volume $V o l$ that contains all the plasma of a given scenario and get, by toroidal symmetry:

$$
\int_{\partial S} \frac{\nabla \psi \cdot \mathbf{n}}{\mu_{0} r} \partial_{t} \psi d s=-\int_{S \cap \Omega_{\mathrm{P}}(\psi)}\left(r p^{\prime}(\psi)+\frac{1}{\mu_{0} r} f f^{\prime}(\psi)\right) \partial_{t} \psi d r d z+\int_{S} \frac{\nabla \psi \cdot \nabla \partial_{t} \psi}{\mu_{0} r} d r d z,
$$

where $S$ denotes the intersection of $V o l$ with the poloidal plane.

If we choose $S$ to be the domain of the plasma $\Omega_{\mathrm{P}}(\psi)$ then the left hand side of equation (3.41) is $V_{\text {loop }} I_{\mathrm{P}}$, where $V_{\text {loop }}$ is the plasma loop voltage. The first integral of the right hand side is related to the time rate of variation of the toroidal magnetic energy and to the work done against the plasma pressure gradient. The last term of the righthand side is the time rate of variation of the poloidal magnetic field energy.

We would like to stress that the integrated poloidal Poynting theorem corresponds to a variational formulation of the Grad-Shafranov-Schlüter equations on $S$. Using the notation (3.2) of our variational formulation from Section 3 we remark that the two integrals on the righthand side correspond to $\mathrm{J}_{\mathrm{p}}\left(\psi, \chi_{S} \partial_{t} \psi\right)$ and $\mathrm{A}\left(\psi, \chi_{S} \partial_{t} \psi\right)$, where $\chi_{S}$ is the characteristic function of $S$. Hence, it can be shown that the solutions $\psi^{k}$ of the evolution problems 15 and 16 fulfill the volume integrated Poynting theorem up to first order accuracy in the mesh size.

The volume integrated version of the toroidal Poynting theorem (2.3) together with the static inverse mode was used in (Ané et al. 2000) for the optimization of ITER scenarios.

\section{Tests and Examples}

\subsection{Validation and Performance}

From the best of our knowledge, there does not exist analytical solutions for the free boundary equilibrium problem considered in this paper. To provide nevertheless some evidence for convergence of the method, we follow a common approach in engineering and study the convergence towards a numerical solution that is computed on a very fine mesh.

We consider a static equilibrium with a given plasma current (Problem 2 ) in ITER geometry. The plasma current is $I_{p}=15.10 \times 10^{6} \mathrm{~A}$ and the current density profile is prescribed using the model 2.11) with $r_{0}=6.2 m \alpha=0.5978, \beta=0.5978, \gamma=1.395$. 

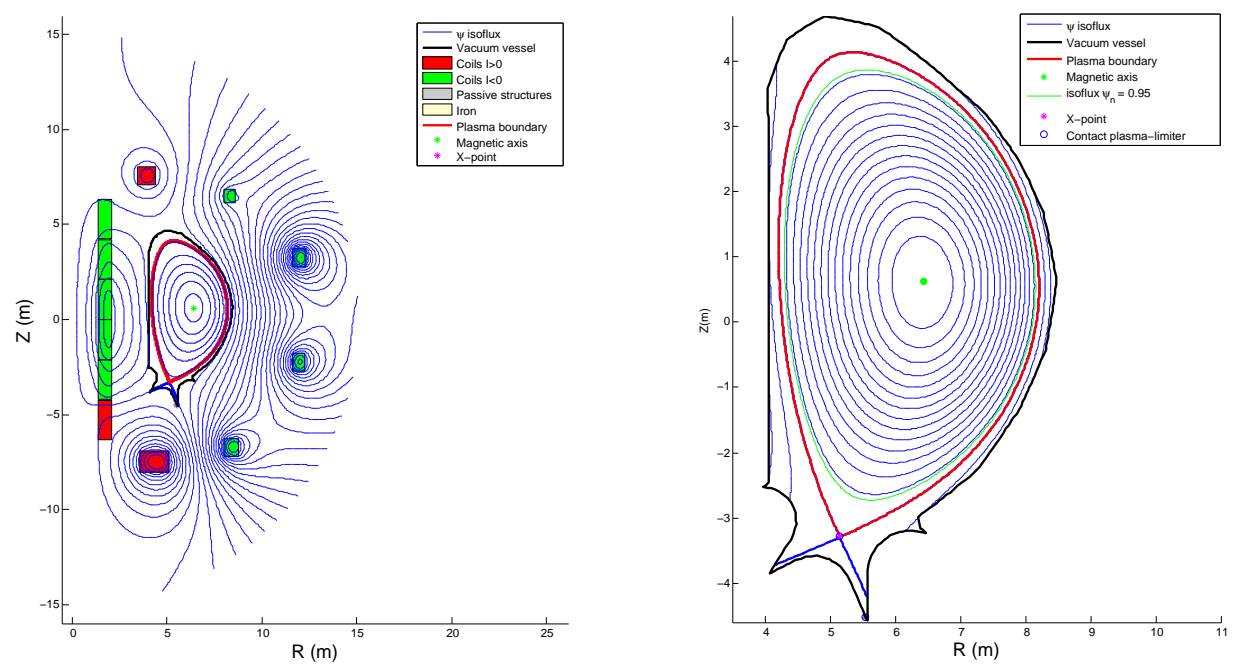

FiguRE 3. The reference solution for the ITER test problem.

With these data we solve the Galerkin Formulation 16 on a sequence of five meshes with increasing number of elements. The solution obtained on the mesh with the largest number of triangular elements, is used as a reference solution and is noted $\psi_{\text {ref }}$. In our case the reference solution $\psi_{\text {ref }}$ has 577415 number of unknowns and the mesh consists of 1153174 triangular elements. The reference solution is depicted in Figure 3 . For each of the other four meshes, the numerical solution $\psi_{N_{u k w n}}$ is evaluated at $N_{\text {points }}=812$ different points of the computational domain which are located independently of the mesh (see Figure 4). Then the relative error

$$
E_{\text {points }}\left(N_{\text {ukwn }}\right)=\frac{\left(\sum_{i=1}^{N_{\text {points }}}\left|\psi_{N_{\text {ukwn }}}\left(M_{i}\right)-\psi_{\text {ref }}\left(M_{i}\right)\right|^{2}\right)^{1 / 2}}{\left(\sum_{i=1}^{N_{\text {points }}}\left|\psi_{\text {ref }}\left(M_{i}\right)\right|^{2}\right)^{1 / 2}}
$$

is used to quantify the convergence. The values in Figure 4 demonstrate the expected linear convergence. Similarly, in Figure 5 we monitor convergence of the plasma quantities total plasma volume $\mathrm{Vol}_{S}$, the numerical derivative $\operatorname{Vol}_{s}^{\prime}$ (see (3.38)) and the geometric coefficient $G_{s}:=\left\langle|\nabla \rho|^{2} r^{-2}\right\rangle_{s}$ (see (3.39) ) that are computed in a post-processing step from the numerical solution $\psi_{h}$ with the methods from Section 3.5. Here again (see Figure 5 we observe approximately linear convergence in the number of unknowns.

In table 2 we give the overall computing time for the previous five computations on an Intel Sandy Bridge 2.6GHz. The computing time scales linearly with the number of unknowns. Given the fact that we solve non-linear problems, the computation time is reasonably small. Application engineers can easily solve a huge amount of different scenarios in short time to do parameter studies for example. CEDRES ++ is perfectly suitable in larger workflow environments, such as the European Integrated Tokamak Modelling Project (Falchetto et al. 2014, ITM|2013). One reason for such short running times is the Newton method. The convergence history of the residuum in Table 2 shows per- 

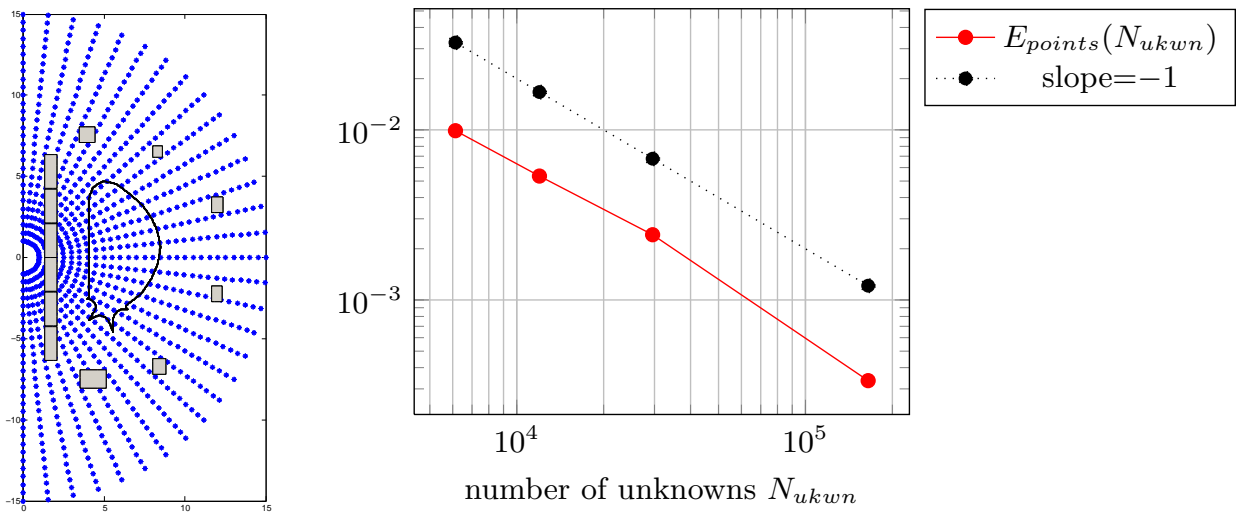

FIgURE 4. Left: the location of the points in the definition of $E_{\text {points }}$. Right: convergence of $E_{\text {point }}$.

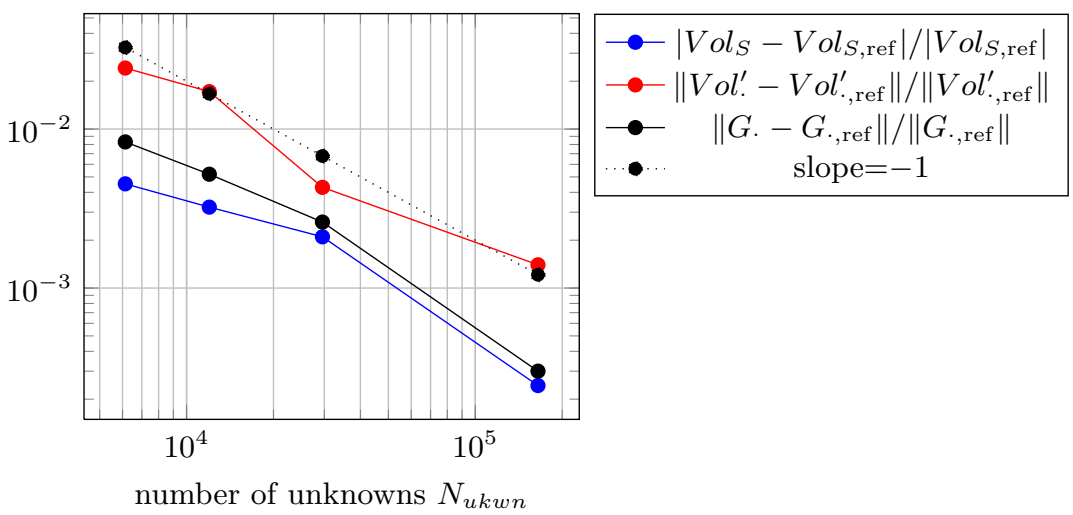

Figure 5. Convergence of plasma volume $\mathrm{Vol}_{S}$, the numerical derivative $V o l_{s}^{\prime}=\left(\frac{\partial V o l}{\partial \rho}\right)_{s}, s=0, \ldots S$ and the geometric coefficient $G_{s}=\left\langle|\nabla \rho|^{2} r^{-2}\right\rangle_{s}, s=0, \ldots S$. The reference quantities $V o l_{S, \text { ref }}, V o l_{s, \text { ref }}^{\prime}$ and $G_{s, \text { ref }}$ correspond to the quantities computed for $\psi_{\text {ref }}$.

|number of triangles $\mid$ number of unknowns $\left|\begin{array}{c}\text { computing } \\ \text { time (in s) }\end{array}\right| \quad \mid$ iteration $\mid$ relative residual |

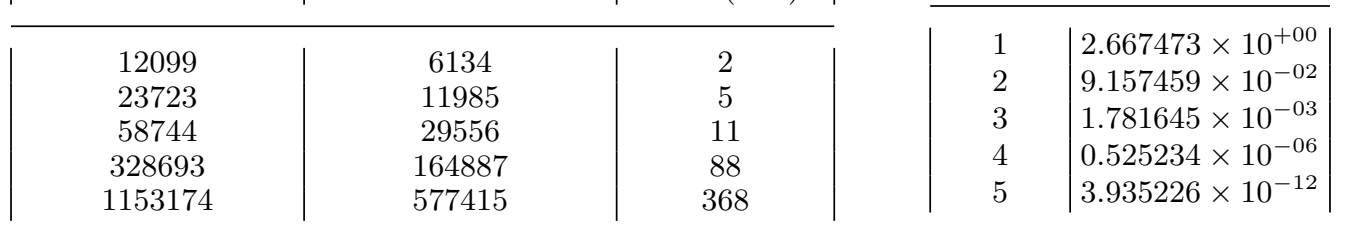

TABLE 2. Left: calculation time. Right: convergence history of the Newton iteration in the calculation of $\psi_{\text {ref }}$.

fect quadratic convergence: we need only very few iterations to find a numerical solution solving the discrete non-linear problem within the limits of machine precision.

\subsection{Quasi-static plasma equilibrium simulations for WEST}

The Tungsten (W) Environment in Steady-state Tokamak (WEST) project (Bucalossi et al. 2011) aims at equipping Tore Supra with an actively cooled tungsten divertor. This 


\begin{tabular}{c|c|c|c||c|c|c|}
\hline & $\left|\mathbf{B}_{\text {pol }}\right|$ & $\left|\mathbf{H}_{\text {pol }}\right|$ & & $\left|\mathbf{B}_{\text {pol }}\right|$ & $\left|\mathbf{H}_{\text {pol }}\right|$ \\
\hline & \begin{tabular}{|c|c|c|c|c|c|}
1 & 0.00 & 0 & 9 & 1.76 & $7.968 \times 10^{3}$ \\
2 & 0.50 & $3.833 \times 10^{2}$ & 10 & 2.06 & $4.821 \times 10^{4}$ \\
3 & 0.70 & $3.982 \times 10^{2}$ & 11 & 2.25 & $1.628 \times 10^{5}$ \\
4 & 0.80 & $4.102 \times 10^{2}$ & 12 & 3.05 & $8.090 \times 10^{5}$ \\
5 & 0.88 & $4.270 \times 10^{2}$ & 13 & 4.05 & $1.588 \times 10^{6}$ \\
6 & 1.00 & $4.703 \times 10^{2}$ & 14 & 6.05 & $3.178 \times 10^{6}$ \\
7 & 1.20 & $6.274 \times 10^{2}$ & 15 & 98.20 & $7.651 \times 10^{7}$ \\
8 & 1.52 & $2.474 \times 10^{3}$ & 16 & $10^{5}$ & $7.957 \times 10^{10}$
\end{tabular}
\end{tabular}

TABLE 3. The data for the poloidal magnetic field $\left|\mathbf{B}_{\text {pol }}\right|$ and the poloidal magnetizing field $\left|\mathbf{H}_{\text {pol }}\right|$ that is used to reconstruct the magnetic permeability.

represents a major change in the magnetic configuration of Tore Supra, moving from a circular limited configuration to a diverted (or X-point) configuration. CEDRES++ is one of the main modeling tools used for the preparation of WEST. It has been employed in particular for the definition of reference equilibria, the dimensioning of the plasma vertical position feedback system, the design of the plasma shape controller, breakdown studies, disruption simulations, etc. We give below a few examples of CEDRES ++ simulations for WEST. Note that Tore Supra is an iron core tokamak and that the iron is taken into account in all of these simulations. The six return arms of the iron core are represented in CEDRES ++ by an axisymmetric equivalent model, which gives the $1 / R$ shape of the return arms visible in Figure 6. We are using the experimental data for the poloidal magnetic field $\mathbf{B}_{\text {pol }}$ and the poloidal magnetizing field $\mathbf{H}_{\text {pol }}$ from table 3 , do piecewise linear interpolation of these data and reconstruct the permeability for arbitrary magnetic field values via $\mu_{\mathrm{FE}}\left(\mathbf{B}_{p o l}^{2}\right)=\left|\mathbf{B}_{\text {pol }}\right|\left|\mathbf{H}_{\text {pol }}\right|^{-1}$.

We present in the following sections three different examples from research for WEST that use the static direct, static evolution and inverse static modes of CEDRES ++ . First simulations with the inverse evolution mode are presented in (Blum \& Heumann 2014). The inverse static mode of CEDRES ++ is also extremely useful in order to define and optimize reference equilibria. We will give details for WEST in a forthcoming publication.

\subsubsection{The current-focused case: direct static mode}

Figure 6 shows a typical WEST poloidal flux map calculated by CEDRES++ in current-focused mode. The X-point is visible at the bottom of the plasma. Here, CEDRES ++ solves the direct static Problem 2 with prescribed total plasma current $I_{\mathrm{P}}=$ $700 k A$ and with the parametrized current profiles $S_{p^{\prime}}$ and $S_{f f^{\prime}}$ in (2.11), using $\alpha=1$, $\beta=1.5, \gamma=0.9, r_{0}=2.6 \mathrm{~m}$. The vacuum toroidal field is $B_{0}=3.524 T$ at $r=2.6 \mathrm{~m}$. A few output parameters are: $\beta_{p}=1.70, l i=0.93, q_{95}=3.33, q_{0}=1.17$.

\subsubsection{The voltage-evolution-focused case, direct evolution mode}

Starting from the equilibrium in the previous section, we run CEDRES ++ in direct evolution mode to solve problem 6. We keep all the input parameters fixed and we apply a constant voltage to the coils, equal to the resistive voltage being the product of coil resistance and current, except for the divertor coils, where we perturb the resistive voltage with $\Delta V=+0.1 \mathrm{Volt}$ in the lower coil and $\Delta V=-0.1 \mathrm{Volt}$ in the upper coil. This is in order to trigger a vertical instability (otherwise the plasma would stay in place). The simulation is run with a time step of $20 \mathrm{~ms}$. Figure 7 shows the plasma boundary at 


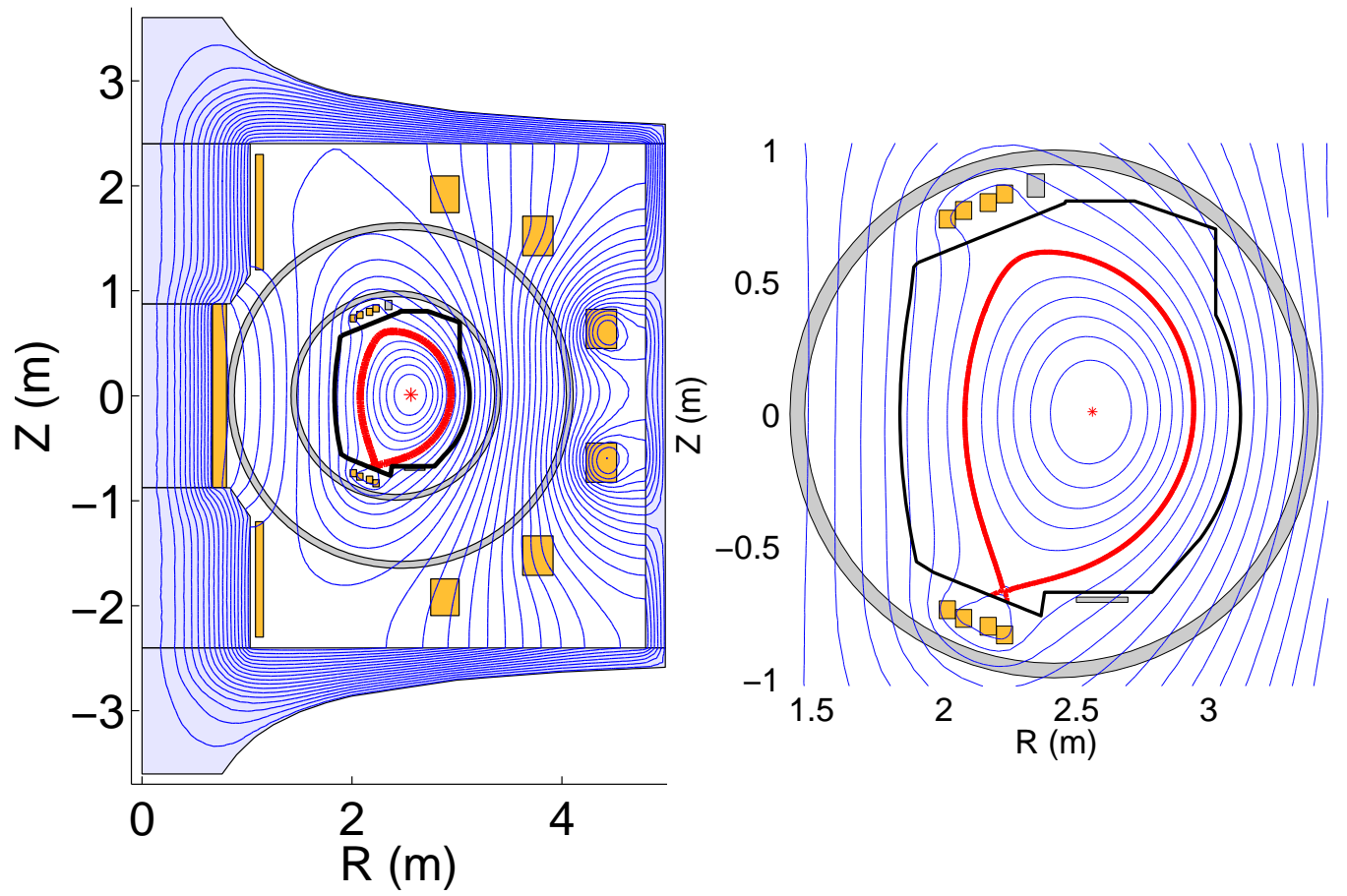

FigURE 6. Poloidal cross section showing $\psi$-isolines for a WEST typical equilibrium. Left: global view; right: zoom on the inner vacuum vessel region. The iron is displayed in blue, the poloidal field coils in orange and the passive structures (vacuum vessel and vertical stabilization components) in grey. The black curve is the limiter curve, i.e. the domain accessible to the plasma. The red curve is the plasma boundary and the red star the magnetic axis.

intervals of $100 \mathrm{~ms}$. The plasma moves down and the diverted configuration is lost after a few $100 \mathrm{~ms}$ when the plasma comes in contact with the baffle of the pumping system. Figure 7 shows the vertical position of the magnetic axis $z_{\mathrm{ax}}$ as a function of time. The early evolution is exponential (as one expects) with a time constant $\tau_{z}=95 \mathrm{~ms}$, while the later evolution is rather linear. The error of the integrated Poynting theorem from section 3.6 is approximately $5 \%$ and decreases if the time step size and the triangle size are refined.

\subsubsection{The current-focused case, inverse static mode}

We present here an example that requires to solve an inverse free-boundary equilibrium problem: the post-processing of CRONOS (Artaud et al. 2010) simulations of WEST scenarios. Indeed, CRONOS simulations are typically run in fixed-boundary equilibrium mode (using the HELENA equilibrium code (Huysmans et al. 1991) with a prescribed boundary geometry). One may however need to know the magnetic field outside the plasma boundary (for example in order to prepare JOREK (Huysmans \& Czarny 2007) non-linear MHD simulations), or to assess whether the scenario is feasible in terms of current limits in the coils for example. These questions may be addressed by solving the inverse Problems 17 and 18 using as desired boundary the boundary used in the CRONOS simulation, and as current profile those profiles $S_{p^{\prime}}$ and $S_{f f^{\prime}}$ that are calculated by CRONOS. These profiles are shown in Figure 8, where one can notice peaks at the edge of the plasma which are characteristic for $\mathrm{H}$-mode profiles, while Figure 8 shows 

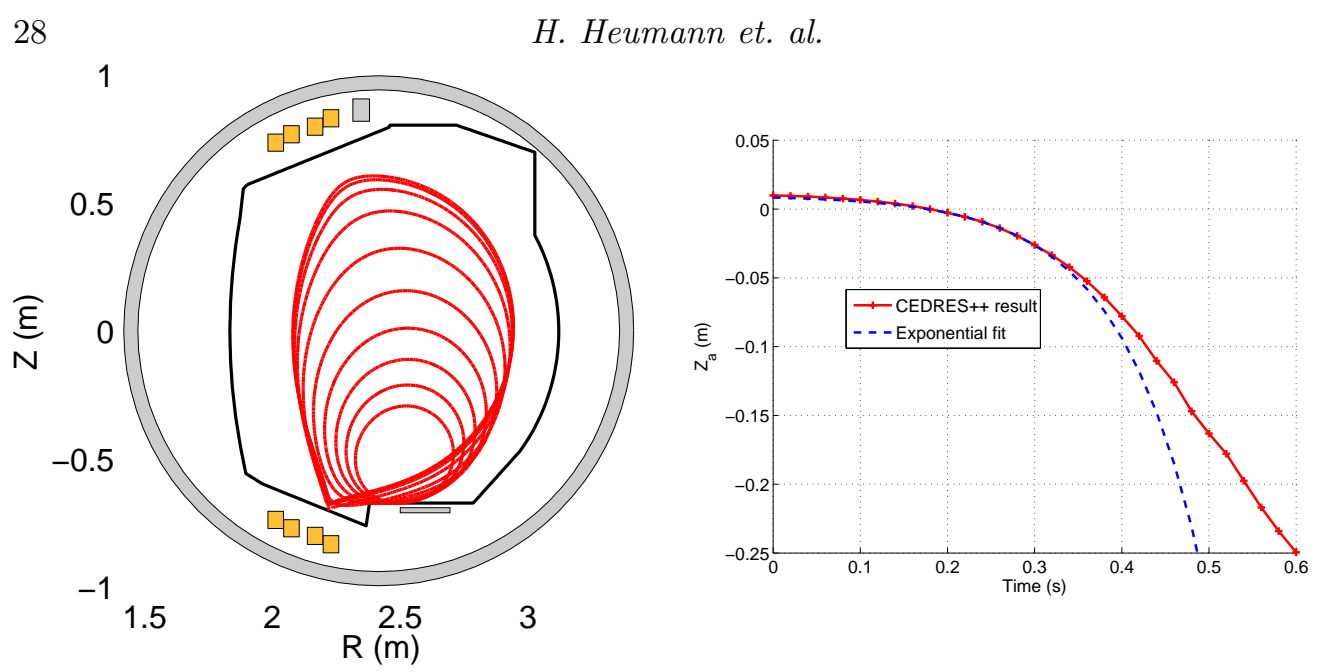

FiguRE 7. Left: Plasma boundary at intervals of $100 \mathrm{~ms}$ in a vertical instability simulation for WEST. Right: Time evolution of the vertical position of the magnetic axis $z_{\text {ax }}$ in a vertical instability simulation for WEST.
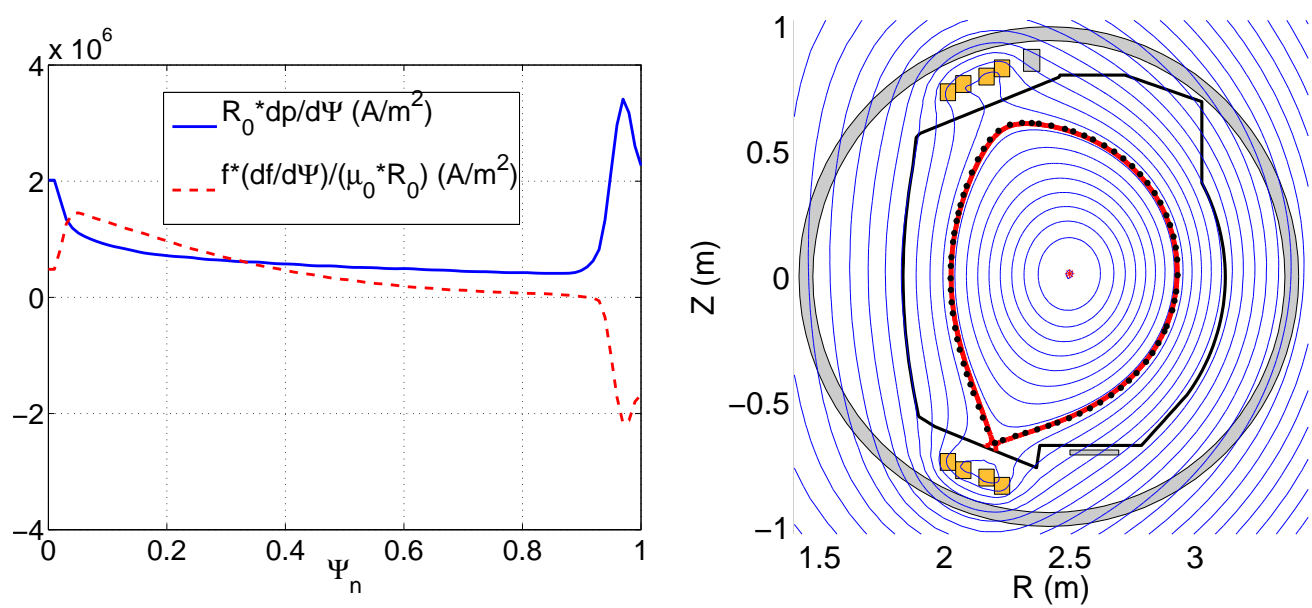

FigurE 8. Left: The profile functions $S_{p^{\prime}}$ and $S_{f f^{\prime}}$ (normalized so as to fit in the same figure) from a CRONOS WEST scenario simulation. Right: Lines: $\psi$-isolines calculated by CEDRES++ in inverse snapshot mode. Black dots: CRONOS plasma boundary, used as the desired plasma boundary in CEDRES++.

the result of the CEDRES++ calculation. It can be seen that the CRONOS boundary (black dots) is well matched.

\section{Conclusions and Perspectives}

We have presented in detail the computational methods of CEDRES++. It enables to compute quasi-static equilibrium configurations, the currents in the poloidal field coils or the voltages applied in the circuits of the poloidal field system being prescribed. In its inverse mode the code computes these currents and voltages that ensure a certain prescribed plasma shape that might evolve in time.

Due to its stability and robustness, CEDRES++ is a perfect tool to be coupled with transport codes (Hinton \& Hazeltine 1976: Hirshman \& Jardin 1979), so that the evo- 
lution of plasma equilibrium is simulated at the resistive timescale consistently with transport processes. Reciprocally the transport codes take into account the precise geometry of the magnetic flux lines. The numerical stability of such a coupling is challenging and subject of ongoing research. This is particularly important for the simulation and optimization of scenarios in new generation tokamaks. CEDRES ++ , when coupled to the transport codes CRONOS (Artaud et al. 2010) and ETS (Coster et al. 2010), is in use for simulating such self consistent plasma evolution. The evolution mode itself, when plasma current profiles are given, is a good practical approach for vertical stability studies where the timescale of interest is much shorter than the current diffusion timescale of the plasma.

Furthermore, the modular and clear structure of CEDRES++ and the emphasis on accurate Newton methods, will make CEDRES++ very useful to implement fully automatic approaches to the optimization of scenarios. It will be easy to study and predict operational limits, and to devise control strategies that circumvent such limits.

It is possible to extend the methods presented in this work to higher order finite elements. Nevertheless, there are a couple of obstacles in order to obtain entirely higher order accurate methods:

- We are solving here a non-linear elliptic problem with discontinuous coefficients (in the case of iron-transformer tokamaks) and discontinuous righthand side. The standard convergence theory for finite elements and elliptic regularity theory does not yield improved approximation results for polynomials of degree higher than 1 . Nevertheless practical experience and the theoretical results in (Feistauer \& Sobotkov 1990) for low-order approximation of non-linear problems and in $(\mathrm{Li}$ et al. $\mid 2010)$ for high-order approximation of linear problems suggest improved accuracy. From the regularity theory for magneto-statics, we know in the case of iron-transformer tokamaks (such as JET or Tore Supra) that the solution lacks of regularity in the vicinity of the iron parts, in particular close to the interfaces air/iron and the corners of subdomains with iron. In such nonregular settings, it is required to switch to the so-called hp-version of the finite element method (Schwab 2004), that uses small triangles and low order polynomials in regions with non-regular solution and large triangles and high order polynomials elsewhere.

- Moreover, the general setting suffers from a fairly large modeling error due to the experimental permeability curve and to the axisymmetric representation of the ferromagnetic circuit that in reality consists of an iron core and a certain number of nonaxisymmetric return limbs. It is not clear whether such modeling errors might surpass the discretization error.

- Higher order accuracy requires also sufficiently accurate quadrature rules in the definition of the Galerkin methods. While such higher order quadrature rules are standard for (iso-parametric) finite elements, we foresee technical difficulties alongside with a considerable increase of computational complexity for the integrals over the intersection of the plasma with triangles. We need to implement sufficiently accurate quadrature rules for polygonal domains with non-straight boundaries. On top of this, we need to implement for the Newton method the derivatives of such quadrature rules.

We are planning to investigate such topics in the near future and to compare with alternative approaches. One promising alternative might be to switch after a couple of Newton iterations to a different discretization scheme that uses separate meshes and separate polynomial degrees for the representation of the flux in the plasma domain and its exterior. The non-linear coupling of the plasma and its exterior will lead to iteration schemes that induce small variations of the two meshes from one iteration to the next. With this, both the location of the magnetic axis and X-point are a priori not limited 
to a finite set of points, as it is the case for linear Lagrangian elements with fixed global mesh.

Any higher order method will involve more sophisticated algorithms and it is hard to predict if the accuracy at a fixed computing time will drastically improve. If high accuracy is required at the moment, this can still be achieved with reasonable effort by simple mesh refinement. The running time of the current version of the code is not yet optimized and still it is possible to do calculations with over half a million unknowns in less than 7 minutes on a workstation.

\section{Acknowledgments}

This work, supported by the European Communities under the contract of Association between EURATOM-CEA was carried out within the framework of the Task Force on Integrated Tokamak Modelling of the European Fusion Development Agreement. The views and opinions expressed herein do not necessarily reflect those of the European Commission.

The authors would like to thank the reviewers for their comments that help improve the manuscript. 


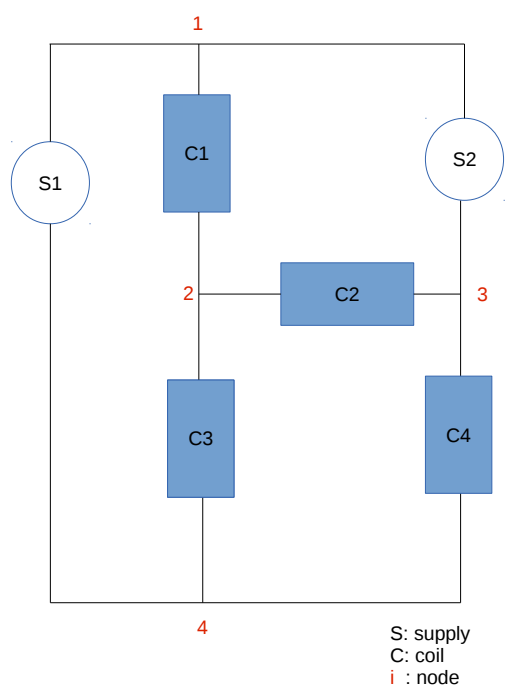

Figure 9. Example of PF-circuit

\section{Appendix A: Circuit equations}

In a tokamak, the poloidal field system is made of a number of circuits each comprising a number of coils and power supplies (see Figure 9). In the following we will present the derivation of the circuit equations for one single circuit.

Let $N_{N}$ be the number of nodes, $N_{C}$ the number of coils, and $N_{S}$ the number of supplies of one circuit. To get a model for this circuit taking into account all those connections, the idea is to write the potential difference for each supply and each coil of the circuit. For a supply $S$, we have

$$
U_{k}-U_{l}=V
$$

where $V$ is the applied voltage and $U_{k}$ and $U_{l}$ the potential at the nodes that enclose the supply. For a coil $C$, the potential difference writes

$$
U_{i}-U_{j}=R \frac{I}{n}+\frac{2 \pi n}{S} \int_{C} \frac{\partial \psi}{\partial t} d r d z
$$

where $U_{i}$ and $U_{j}$ are the potentials at the nodes that enclose the coil, $R$ is the total resistance of the coil $C, S$ its cross section, $n$ its number of wire turns and $I$ the total current (in Ampère-turns). The average of $\frac{\partial \psi}{\partial t}$ over the coil $C$ is an approximation of the discrete sum of inductive terms seen by the various turns of the coil. This approximation is perfectly valid, if one has a homogeneous distribution of turns in the coil.

We also consider Kirchhoff's current law at each node of the circuit adding $N_{N}$ equations to the system. To fix the potential, we suppose that $U_{1}=0$. Thus, we get a set of $N_{e q}=N_{S}+N_{C}+N_{N}+1$ equations which can be written in the form

$$
\boldsymbol{A} \vec{U}=\boldsymbol{B} \vec{V}+\boldsymbol{C} \vec{I}+\boldsymbol{D} \vec{\Psi}\left(\partial_{t} \psi\right) .
$$

where the $N_{S}$ first equations represent (6.1) and the following $N_{C}$ equations are 6.2). The last equation of the system fixes the potential $U_{1}$. The matrices $\boldsymbol{A} \in \mathbb{R}^{N_{e q} \times N_{N}}$, 
$\boldsymbol{B} \in \mathbb{R}^{N_{e q} \times N_{S}}, \boldsymbol{C} \in \mathbb{R}^{N_{e q} \times\left(N_{S}+N_{C}\right)}$ and $\boldsymbol{D} \in \mathbb{R}^{N_{e q} \times N_{C}}$ are called potential matrix, voltage matrix, current matrix and induction matrix, respectively. The vectors $\vec{U} \in \mathbb{R}^{N_{N}}$, $\vec{V} \in \mathbb{R}^{N_{S}}$ and $\vec{I} \in \mathbb{R}^{N_{S}+N_{C}}$ contain the electric potential at the circuit nodes, the voltages applied at the supplies and currents at the coils and the supplies. The components of the vector $\vec{\Psi}\left(\partial_{t} \psi\right) \in \mathbb{R}^{N_{C}}$ are the integrals $\int_{C} \partial_{t} \psi d r d z$ over the domain that is occupied by a coil $C$ of the circuit.

For given $\vec{V}, \vec{I}$ and $\vec{\Psi}\left(\partial_{t} \psi\right)$, there is a unique $\vec{U}$ which satisfies $(6.3)$, hence $\boldsymbol{A}^{T} \boldsymbol{A}$ is regular. We find

$$
\vec{U}=\left(\boldsymbol{A}^{T} \boldsymbol{A}\right)^{-1} \boldsymbol{A}^{T} \boldsymbol{B} \vec{V}+\left(\boldsymbol{A}^{T} \boldsymbol{A}\right)^{-1} \boldsymbol{A}^{T} \boldsymbol{C} \vec{I}+\left(\boldsymbol{A}^{T} \boldsymbol{A}\right)^{-1} \boldsymbol{A}^{T} \boldsymbol{D} \vec{\Psi}\left(\partial_{t} \psi\right),
$$

plug this into 6.3 and get

$$
\boldsymbol{E} \vec{I}+\boldsymbol{F} \vec{V}+\boldsymbol{G} \vec{\Psi}\left(\partial_{t} \psi\right)=0
$$

with

$$
\begin{aligned}
& \boldsymbol{E}=\boldsymbol{A}\left(\boldsymbol{A}^{T} \boldsymbol{A}\right)^{-1} \boldsymbol{A}^{T} \boldsymbol{C}-\boldsymbol{C} \\
& \boldsymbol{F}=\boldsymbol{A}\left(\boldsymbol{A}^{T} \boldsymbol{A}\right)^{-1} \boldsymbol{A}^{T} \boldsymbol{B}-\boldsymbol{B} \\
& \boldsymbol{G}=\boldsymbol{A}\left(\boldsymbol{A}^{T} \boldsymbol{A}\right)^{-1} \boldsymbol{A}^{T} \boldsymbol{D}-\boldsymbol{D} .
\end{aligned}
$$

The system (6.5) of $N_{e q}$ equations is over determined and $\vec{I}$ can be computed using the normal equation

$$
\vec{I}=\boldsymbol{S} \vec{V}+\boldsymbol{R} \vec{\Psi}\left(\partial_{t} \psi\right)
$$

with $\boldsymbol{S}=-\left(\boldsymbol{E}^{T} \boldsymbol{E}\right)^{-1} \boldsymbol{E}^{T} \boldsymbol{F}$ and $\boldsymbol{R}=-\left(\boldsymbol{E}^{T} \boldsymbol{E}\right)^{-1} \boldsymbol{E}^{T} \boldsymbol{G}$. 


\section{REFERENCES}

Albanese, R., Blum, J. \& Barbieri, O. 1986 On the solution of the magnetic flux equation in an infinite domain. In EPS. 8th Europhysics Conference on Computing in Plasma Physics (1986).

Albanese, R., Blum, J. \& De Barbieri, O. 1987 Numerical studies of the Next European Torus via the PROTEUS code. In 12th Conf. on Numerical Simulation of Plasmas, San Francisco.

Albanese, R. \& Villone, F. 1998 The linearized create-l plasma response model for the control of current, position and shape in tokamaks. Nuclear Fusion 38 (5), 723.

Ané, J.M., Grandgirard, V., Albajar, F. \& Johner, J. 2000 Design of next step tokamak: Consistent analysis of plasma performance flux composition and poloidal field system. In 18th IAEA Fusion Energy Conference, Sorrento, http: //www. iaea. org/inis/ collection/NCLCollectionStore/_Public/33/029/33029055. pdf

Ariola, M. \& Pironti, A. 2008 Magnetic Control of Tokamak Plasmas. Springer London.

Artaud, J.F., Basiuk, V., Imbeaux, F., Schneider, M., Garcia, J., Giruzzi, G., Huynh, P., Aniel, T., Albajar, F., Ané, J.M., Bécoulet, A., Bourdelle, C., Casati, A., Colas, L., Decker, J., Dumont, R., Eriksson, L.G., Garbet, X., Guirlet, R., Hertout, P., Hoang, G.T., Houlberg, W., Huysmans, G., Joffrin, E., Kim, S.H., Kchl, F., Lister, J., Litaudon, X., Maget, P., Masset, R., Pégourié, B., Peysson, Y., Thomas, P., Tsitrone, E. \& Turco, F. 2010 The CRONOS suite of codes for integrated tokamak modelling. Nuclear Fusion 50 (4), 043001.

Bielak, J. \& MACCAMY, R. C. 1991 Symmetric finite element and boundary integral coupling methods for fluid-solid interaction. Quart. Appl. Math. 49 (1), 107-119.

Blum, J. 1987 Numerical simulation of the plasma equilibrium in a tokamak. Wiley/GauthierVillars.

Blum, J., Boulbe, C. \& Faugeras, B. 2012 Reconstruction of the equilibrium of the plasma in a tokamak and identification of the current density profile in real time. Journal of Computational Physics 231 (3), 960 - 980.

Blum, J. \& Heumann, H. 2014 Optimal control for quasi-static evolution of plasma equilibrium in tokamaks. Tech. Rep.. INRIA, Sophia Antipolis.

Blum, J., Le Foll, J. \& Thooris, B. 1981 The self-consistent equilibrium and diffusion code SCED. Computer Physics Communications 24, 235 - 254.

Bucalossi, J., Argouarch, A., Basiuk, V., Baulaigue, O., Bayetti, P., Bcoulet, M., Bertrand, B., Brmond, S., Cara, P., Chantant, M., Corre, Y., Courtois, X., DoCeul, L., Ekedahl, A., Faisse, F., Firdaouss, M., Garcia, J., Gargiulo, L., Gil, C., Grisolia, C., Gunn, J., Hacquin, S., Hertout, P., Huysmans, G., Imbeaux, F., Jiolat, G., Johnny, M., Jourd'heuil, L., Jouve, M., Kukushkin, A., Lipa, M., Lisgo, S., Loarer, T., Maget, P., Magne, R., Marandet, Y., Martinez, A., Mazon, D., Meyer, O., Missirlian, M., Monier-Garbet, P., Moreau, P., Nardon, E., Panayotis, S., Pgouri, B., Pitts, R.A., Portafaix, C., Richou, M., Sabot, R., Saille, A., Saint-Laurent, F., Samaille, F., Simonin, A. \& Tsitrone, E. 2011 Feasibility study of an actively cooled tungsten divertor in tore supra for ITER technology testing. Fusion Engineering and Design 86 (6-8), $684-688$.

Chen, Goong \& Zhou, Jianxin 1992 Boundary element methods. Academic Press, Ltd., London.

Ciarlet, P. G. 1978 The Finite Element Method for Elliptic Problems. Amsterdam: NorthHolland Publishing Co., studies in Mathematics and its Applications, Vol. 4.

Cooper, W.A., Hirshman, S.P., Merkel, P., Graves, J.P., Kisslinger, J., Wobig, H.F.G., Narushima, Y., Okamura, S. \& Watanabe, K.Y. 2009 Three-dimensional anisotropic pressure free boundary equilibria. Computer Physics Communications 180 (9), $1524-1533$.

Costabel, M. \& Stephan, E.P. 1990 Coupling of finite and boundary element methods for an elastoplastic interface problem. SIAM J. Numer. Anal. 27 (5), 1212-1226.

Coster, D.P., Basiuk, V., Pereverzev, G., Kalupin, D., Zagrksi, R., Stankiewicz, R., Huynh, P. \& Imbeaux, F. 2010 The european transport solver. Plasma Science, IEEE Transactions on 38 (9), 2085-2092.

DAVIS, T.A. 2011 Umfpack http://www.cise.ufl.edu/research/sparse/umfpack/ 
Degtyarev, L.M. \& Drozdov, V.V. 1985 An inverse variable technique in the MHDequilibrium problem. Computer Physics Reports 2 (7), 341 - 387.

Degtyarev, L.M. \& Drozdov, V.V. 1991 Adaptive mesh computation of magnetic hydrodynamic equilibrium. International Journal of Modern Physics C 02 (01), 30-38.

Delfour, M.C. \& ZolÉsio, J.-P. 2011 Shapes and geometries, 2nd edn., Advances in Design and Control, vol. 22. Society for Industrial and Applied Mathematics (SIAM), Philadelphia, PA.

DeLucia, J., Jardin, S.C. \& Todd, A.M.M. 1980 An iterative metric method for solving the inverse tokamak equilibrium problem. Journal of Computational Physics 37 (2), 183 - 204.

Falchetto, G.L., Coster, D., Coelho, R., Scott, B.D., Figini, L., Kalupin, D., Nardon, E., Nowak, S., Alves, L.L., Artaud, J.F., Basiuk, V., Bizarro, Joo P.S., Boulbe, C., Dinklage, A., Farina, D., Faugeras, B., Ferreira, J., Figueiredo, A., Huynh, Ph., Imbeaux, F., Ivanova-Stanik, I., Jonsson, T., Klingshirn, H.-J., Konz, C., Kus, A., Marushchenko, N.B., Pereverzev, G., Owsiak, M., Poli, E., Peysson, Y., Reimer, R., Signoret, J., Sauter, O., Stankiewicz, R., Strand, P., Voitsekhovitch, I., Westerhof, E., Zok, T., Zwingmann, W., Contributors, ITMTF, the ASDEX Upgrade Team \& Contributors, JET-EFDA 2014 The European Integrated Tokamak Modelling (ITM) effort: achievements and first physics results. Nuclear Fusion 54 (4), 043018.

Feistauer, Miloslav \& Sobotkov, Veronika 1990 Finite element approximation of nonlinear elliptic problems with discontinuous coefficients. ESAIM: Mathematical Modelling and Numerical Analysis - Modlisation Mathmatique et Analyse Numrique 24 (4), 457-500.

Feneberg, W. \& Lackner, K. 1973 Multipole tokamak equilibria. Nuclear Fusion 13 (4), 549.

Fitzgerald, M., Appel, L.C. \& Hole, M.J. 2013 EFIT tokamak equilibria with toroidal flow and anisotropic pressure using the two-temperature guiding-centre plasma. Nuclear Fusion $53(11), 113040$.

FreidberG, J. P. 1987 Ideal Magnetohydrodynamics. Plenum US.

GaticA, G.N. \& HsiaO, G.C. 1995 The uncoupling of boundary integral and finite element methods for nonlinear boundary value problems. J. Math. Anal. Appl. 189 (2), 442-461.

Glowinski, R. \& Marrocco, A. 1974 Analyse numérique du champ magnétique d'un alternateur par éléments finis et sur-relaxation ponctuelle non linéaire. Comput. Methods Appl. Mech. Engrg. 3 (1), 55-85.

Goedbloed, J.P. \& Lifschitz, A. 1997 Stationary symmetric magnetohydrodynamic flows. Physics of Plasmas (1994-present) 4 (10), 3544-3564.

Goedbloed, Johan P, Keppens, Rony \& Poedts, StefaAn 2010 Advanced magnetohydrodynamics: with applications to laboratory and astrophysical plasmas. Cambridge University Press.

Goedbloed, Johan Peter \& Poedts, StefaAn 2004 Principles of magnetohydrodynamics: with applications to laboratory and astrophysical plasmas. Cambridge university press.

Grad, H. 1967 Toroidal containment of a plasma. Physics of Fluids (1958-1988) 10 (1), 137154.

Grad, H. \& Rubin, H. 1958 Hydromagnetic equilibria and force-free fields. Proceedings of the 2nd UN Conf. on the Peaceful Uses of Atomic Energy 31, 190.

Grandgirard, Virginie 1999 Modélisation de l'équilibre d'un plasma de tokamak. PhD thesis, l'Université de Franche-Comté.

Gruber, R., Iacono, R. \& Troyon, F. 1987 Computation of MHD equilibria by a quasiinverse finite hybrid element approach. Journal of Computational Physics 73 (1), 168 182.

Guazzotto, L., Betti, R., Manickam, J. \& Kaye, S. 2004 Numerical study of tokamak equilibria with arbitrary flow. Physics of Plasmas (1994-present) 11 (2), 604-614.

HeLton, F.J. \& WANG, T.S. 1978 MHD equilibrium in non-circular tokamaks with field-shaping coil systems. Nuclear Fusion 18 (11), 1523.

Hertout, P., Boulbe, C., Nardon, E., Blum, J., Bremond, S., Bucalossi, J., Faugeras, B., Grandgirard, V. \& Moreau, P. 2011 The cedres++ equilibrium code and its application to ITER, JT-60SA and Tore Supra. Fusion Engineering and Design 86, 1045 1048. 
Hinton, F.L. \& Hazeltine, R.D. 1976 Theory of plasma transport in toroidal confinement systems. Rev. Mod. Phys. 48, 239-308.

Hiptmair, R. 2003 Coupling of finite elements and boundary elements in electromagnetic scattering. SIAM J. Numer. Anal. 41 (3), 919-944.

Hirshman, S.P. \& Betancourt, O. 1991 Preconditioned descent algorithm for rapid calculations of magnetohydrodynamic equilibria. J. Comput. Phys. 96 (1), 99-109.

Hirshman, S. P. \& JARDin, S. C. 1979 Two-dimensional transport of tokamak plasmas. Physics of Fluids 22 (4), 731-742.

Hofmann, F. \& Tonetti, G. 1988 Tokamak equilibrium reconstruction using Faraday rotation measurements. Nuclear Fusion 28 (10), 1871.

Huysmans, G.T.A. \& Czarny, O. 2007 MHD stability in X-point geometry: simulation of ELMs. Nuclear Fusion 47 (7), 659.

Huysmans, G.T.A., Goedbloed, J.P. \& Kerner, W. 1991 Isoparametric bicubic Hermite elements for solution of the Grad-Shafranov equation. Proc. CP90 Conf. on Comp. Phys. p. 371.

ITM 2013 Integrated tokamak modelling. http://portal.efda-itm.eu/, integrated Tokamak Modelling.

JARDIN, S.C. 2010 Computational methods in plasma physics. Boca Raton, FL : CRC Press/Taylor \& Francis.

Jardin, S. C., Pomphrey, N. \& Delucia, J. 1986 Dynamic modeling of transport and positional control of tokamaks. J. Comput. Phys. 66, 481-507.

Johnson, J.L., Dalhed, H.E., Greene, J.M., Grimm, R.C., Hsieh, Y.Y., Jardin, S.C., Manickam, J., Okabayashi, M., Storer, R.G., Todd, A.M.M., Voss, D.E. \& WeImer, K.E. 1979 Numerical determination of axisymmetric toroidal magnetohydrodynamic equilibria. Journal of Computational Physics 32 (2), 212 - 234.

LaCKner, K. 1976 Computation of ideal MHD equilibria. Computer Physics Communications $12(1), 33-44$.

Lao, L.L., Ferron, J.R., Geoebner, R.J., Howl, W., St. John, H.E., Strait, E.J. \& TAYLOR, T.S. 1990 Equilibrium analysis of current profiles in Tokamaks. Nuclear Fusion $30(6), 1035$.

LaO, L.L., Hirshman, S.P. \& Wieland, R.M. 1981 Variational moment solutions to the Grad-Shafranov equation. Physics of Fluids 24 (8), 1431-1440.

Lao, L.L., John, H. St., Stambaugh, R.D., Kellman, A.G. \& Pfeiffer, W. 1985 Reconstruction of current profile parameters and plasma shapes in tokamaks. Nuclear Fusion 25 (11), 1611.

Li, Jingzhi, Melenk, Jens Markus, Wohlmuth, Barbara \& Zou, Jun 2010 Optimal a priori estimates for higher order finite elements for elliptic interface problems. Applied Numerical Mathematics 60 (1-2), 19 - 37.

Ling, K.M. \& JARdin, S.C. 1985 The Princeton spectral equilibrium code: PSEC. Journal of Computational Physics 58 (3), 300 - 335.

Lüst, R. \& Schlüter, A. 1957 Axialsymmetrische magnetohydrodynamische Gleichgewichtskonfigurationen. Z. Naturforsch. A 12, 850-854.

Luxon, J.L. \& Brown, B.B. 1982 Magnetic analysis of non-circular cross-section tokamaks. Nuclear Fusion 22 (6), 813.

Maschke, E.K. \& Perrin, H.J. 1984 An analytic solution of the stationary $\{$ MHD $\}$ equations for a rotating toroidal plasma. Physics Letters A 102 (3), 106 - 108.

Mc Carthy, P.J., Martin, P. \& Schneider, W. 1999 The CLISTE Interpretive Equilibrium Code. Tech. Rep. IPP Report 5/85. Max-Planck-Institut fur Plasmaphysik.

Murat, F. \& Simon, J. 1976 Sur le contrôle par un domaine géométrique. Tech. Rep. 76015. Laboratoire d'Analyse Numérique, Université de Paris 6.

NÉDÉLEC, J.-C. 2001 Acoustic and electromagnetic equations, Applied Mathematical Sciences, vol. 144. Springer-Verlag, New York.

Nocedal, J. \& Wright, S. J. 2006 Numerical optimization, 2nd edn. New York: Springer.

Parail, V., Albanese, R., Ambrosino, R., Artaud, J.-F., Besseghir, K., Cavinato, M., Corrigan, G., Garcia, J., Garzotti, L., Gribov, Y., Imbeaux, F., Koechl, F., Labate, C.V., Lister, J., Litaudon, X., Loarte, A., Maget, P., Mattei, M., McDonald, D., Nardon, E., Saibene, G., Sartori, R. \& Urban, J. 2013 Self-consistent 
simulation of plasma scenarios for iter using a combination of $1.5 \mathrm{~d}$ transport codes and free-boundary equilibrium codes. Nuclear Fusion 53 (11), 113002.

Park, W., Belova, E. V., Fu, G. Y., Tang, X. Z., Strauss, H. R. \& Sugiyama, L. E. 1999 Plasma simulation studies using multilevel physics models. Physics of Plasmas (1994present) 6 (5), 1796-1803.

Pechstein, C. \& Jüttler, B. 2006 Monotonicity-preserving interproximation of $B$ - $H$-curves. J. Comput. Appl. Math. 196 (1), 45-57.

Pustovitov, V.D. 2010 Anisotropic pressure effects on plasma equilibrium in toroidal systems. Plasma Physics and Controlled Fusion 52 (6), 065001.

Renard, Y. \& Pommier, J. 2014 GetFEM++, an open-source finite element library. http: //download.gna.org/getfem/html/homepage/index.html

SCHWAB, C. $2004 p$ - and $h p$ - finite element methods : theory and applications in solid and fluid mechanics. Oxford : Clarendon Press.

Shafranov, V.D. 1958 On magnetohydrodynamical equilibrium configurations. Soviet Journal of Experimental and Theoretical Physics 6, 545.

Shewchuk, J.R. 1996 Triangle: Engineering a 2D Quality Mesh Generator and Delaunay Triangulator. In Applied Computational Geometry: Towards Geometric Engineering (ed. Ming C. Lin \& Dinesh Manocha), Lecture Notes in Computer Science, vol. 1148, pp. 203-222. Springer-Verlag, from the First ACM Workshop on Applied Computational Geometry.

Stephan, E.P. 1992 Coupling of finite elements and boundary elements for some nonlinear interface problems. Comput. Methods Appl. Mech. Engrg. 101 (1-3), 61-72.

Turkington, B., Lifschitz, A., Eydeland, A. \& Spruck, J. 1993 Multiconstrained variational problems in magnetohydrodynamics: Equilibrium and slow evolution. Journal of Computational Physics 106 (2), 269 - 285.

Wesson, J. 2004 Tokamaks. Oxford University Press.

ZhaO, K., Vouvakis, M.N. \& LeE, J.-F. 2006 Solving electromagnetic problems using a novel symmetric fem-bem approach. Magnetics, IEEE Transactions on 42 (4), 583-586.

Zwingmann, W., Eriksson, L.-G. \& Stubberfield, P. 2001 Equilibrium analysis of tokamak discharges with anisotropic pressure. Plasma Physics and Controlled Fusion 43 (11), 1441. 